\title{
Cabibbo suppressed single pion production off the nucleon induced by antineutrinos
}

\author{
M. Benitez Galan $\odot,{ }^{1, *}$ M. Rafi Alam $\oplus^{2, \dagger}$ and I. Ruiz Simo $\oplus^{3, \dagger}$ \\ ${ }^{1}$ Departamento de Física Atómica, Molecular y Nuclear, Facultad de Ciencias, \\ Universidad de Granada, E-18071 Granada, Spain \\ ${ }^{2}$ Department of Physics, Aligarh Muslim University, Aligarh-202 002, India \\ ${ }^{3}$ Departamento de Física Atómica, Molecular y Nuclear and Instituto Interuniversitario Carlos I de Física \\ Teórica y Computacional, Facultad de Ciencias, Universidad de Granada, E-18071 Granada, Spain
}

(Received 17 August 2021; accepted 23 September 2021; published 19 October 2021)

\begin{abstract}
In this work we study the $\Sigma \pi$ and $\Lambda \pi$ production off free nucleons driven by the strangeness-changing weak charged current. We calculate the total cross sections for all possible channels and estimate the fluxaveraged total cross sections for experiments like MiniBooNE, SciBooNE, T2K, and Minerva. The model is based on the lowest order effective SU(3) chiral Lagrangians in the presence of an external weak charged current and contains Born and the lowest-lying decuplet resonant mechanisms that can contribute to these reaction channels. We also compare and discuss our results with others following similar and very different approaches.
\end{abstract}

DOI: 10.1103/PhysRevD.104.073005

\section{INTRODUCTION}

The neutrino and antineutrino-nucleus cross sections are necessary inputs for the analyses of the neutrino scattering and oscillation experiments [1-5]. One of the main ingredients in the (anti)neutrino-nucleus cross sections is the primary (anti)neutrino-nucleon interaction model. It is very important that these models provide accurate predictions when compared with experimental data on nucleon targets, before embedding these elementary interactions within the nuclear medium, where relevant nuclear effects may distort the final signal in experiments. In the few $\mathrm{GeV}$ energy regions, where most of the present [6-8] and future [9-11] oscillation experiments take data, single pion production channels may play a crucial role.

The Cabibbo enhanced single pion production off nucleons is a long-standing theoretical process that has been studied [12-38] and measured [39-61] since many decades ago up to date. However, its Cabibbo suppressed counterpart, where a pion is produced along with a $S=-1$ hyperon $(\Sigma$ or $\Lambda)$ in the final state, is a scarcely studied set of reactions.

\footnotetext{
*mbenitezgalan@ugr.es

rafi.alam.amu@gmail.com

${ }^{\ddagger}$ ruizsig@ugr.es
}

Published by the American Physical Society under the terms of the Creative Commons Attribution 4.0 International license. Further distribution of this work must maintain attribution to the author(s) and the published article's title, journal citation, and DOI. Funded by SCOAP.
In the previous works [62-65], different approaches have been followed. In Ref. [62] a coupled-channel chiral unitary approach is used to dynamically generate the $\Lambda(1405)$ resonance, which plays a major role in the $\pi \Sigma$ reaction channel. In Refs. [63,64] a nonrelativistic 3-quark model, effective $V-A$ theory with experimental form factors, and the relativistic quark model with harmonic interaction of Feynman, Kislinger, and Ravndal [66] are used to calculate the cross section for $\Sigma^{* 0}(1385)$ resonance production off proton, among other channels. Finally, in Ref. [65] a model with background or Born terms is used to calculate a plethora of reactions producing strange particles, in particular the $\pi Y$ production channel, but explicitly excluding $N^{*}$ and $Y^{*}$ exchange mechanisms.

The kind of reactions studied in this work can only be induced by antineutrinos, due to the selection rule for the strangeness-changing weak charged current, $\Delta S=$ $\Delta Q=-1$, for the hadrons. Given that the strangenesschanging weak current changes an $u$ quark into a $s$ quark (or a $\bar{s}$ antiquark into an $\bar{u}$ one), there are also the selection rules $\Delta I=\frac{1}{2}$ and $\Delta I_{z}=-\frac{1}{2}=\frac{\Delta Q}{2}$, where $\left(I, I_{z}\right)$ are the strong isospin and its third component.

Though the present work centered around strangeness changing pion production, the hyperon produced in the final state holds an added advantage. For instance, the inclusive hyperon $(\Lambda$ or $\Sigma$ ) production below the energy threshold for associated $K Y$ production is going to be dominated by the quasielastic (QE) hyperon production channel $[63,67-71]$ and by the reactions studied in this work. In particular, the direct $\Sigma^{+}$production in QE hyperon reactions off nucleons is not allowed; the final appearance 
of $\Sigma^{+}$particles in reactions taking place off nuclear targets is due to the final-state interactions (FSI) or re-scattering experienced by the other hyperons inside the nucleus $[67,69,70]$. However, in the inelastic $(\Delta S=-1)$ channel, $\Sigma^{+}$can be produced in primary antineutrino interaction off protons(for a complete list of final states, please see Sec. II), which is expected to be dominant source of $\Sigma^{+}$production below the $K Y$ threshold. Also, a direct consequence of FSI and nuclear effects is the absorption of produced (primary) pions on a large scale; however, the secondary pions produced from hyperon decay will not suffer a strong absorption thanks to the long lifetime of hyperons.

In this work, we developed a model for (anti)neutrinoinduced $\pi Y$ production on the nucleon induced by the charged current interactions. The present model is largely based on the models that have been well tested in the past, like in $K$-production [72-74], $\pi$-production [27] etc. While the nonresonant mechanism relies on the chiral Lagrangian and $\mathrm{SU}(3)$ flavor symmetry, the resonant mechanism involves both nonstrange $[\Delta(1232)]$ and strange $\left[\Sigma^{*}(1385)\right]$ resonances.

The structure of this work is as follows: in Sec. II we discuss the formalism in detail; in Sec. III we present our results; and finally, in Sec. IV we summarize our findings.

\section{FORMALISM}

In this work we are interested in the following set of antineutrino induced reactions

$$
\bar{\nu}_{l}(k)+N(p) \rightarrow l^{+}\left(k^{\prime}\right)+\pi\left(p_{m}\right)+Y\left(p_{Y}\right),
$$
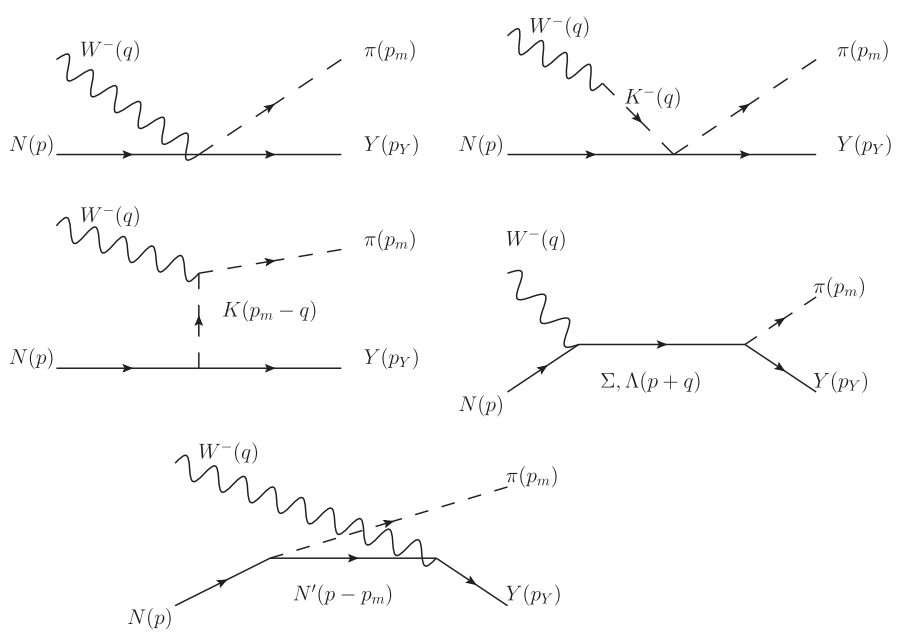

(a) Background or Born terms of our model. From top to bottom and from left to right, we find the contact term (CT), the kaon pole $(\mathrm{KP})$, the kaon-in-flight (KF), the s-channel $\Sigma$ and $\Lambda$ (s- $\Sigma$ and s- $\Lambda$ ) and the u-channel $N(\mathrm{u}-N)$ diagrams, respectively. where $N$ can be either a proton or neutron, $Y$ is a $\Sigma$ or $\Lambda$ hyperon, and the four-momenta of particles are given in parentheses. For induced reactions off protons, the allowed $Y \pi$ final states are $\Lambda \pi^{0}, \Sigma^{0} \pi^{0}, \Sigma^{+} \pi^{-}$, and $\Sigma^{-} \pi^{+}$; while for the neutron channel the possibilities are $\Lambda \pi^{-}, \Sigma^{0} \pi^{-}$, and $\Sigma^{-} \pi^{0}$.

Our model, shown in Fig. 1, is very similar to that of Ref. [65], but also includes the lowest lying decuplet resonances like $\Delta(1232)$ and $\Sigma^{*}(1385)$ as explicit degrees of freedom [shown in Fig. 1(b)], in the line of previous works such as those of Refs. [72-75]. We use effective $V-A$ strangeness-changing weak charged current with vector and axial-vector form factors for the $N-Y^{\prime}$ transitions. The vector form factors are related to the electromagnetic nucleon form factors using the Cabibbo theory, i.e., assuming that the strangeness-changing weak vector current belongs to an SU(3) octet of flavor currents. For the axial-vector currents, $D$-type (symmetric) and $F$-type (antisymmetric) couplings arise between two octets $\{8\} \otimes$ $\{8\}$ that are connected through a SU(3) octet axial current. Whereas, the $q^{2}$-dependence is introduced by assuming a similar form for both $D$ and $F$ couplings, taken to be of dipole form $[67,75]$. For the $\pi N N^{\prime}$ and $\pi Y Y^{\prime}$ strong vertices we assume pseudovector couplings with the derivative of the pseudoscalar meson field. These assumptions are fully consistent with the lowest order baryon-meson chiral Lagrangians in the presence of a weak charged external current, as discussed in [76].

\section{A. Total cross section}

The unpolarized differential cross section corresponding to Eq. (1) is

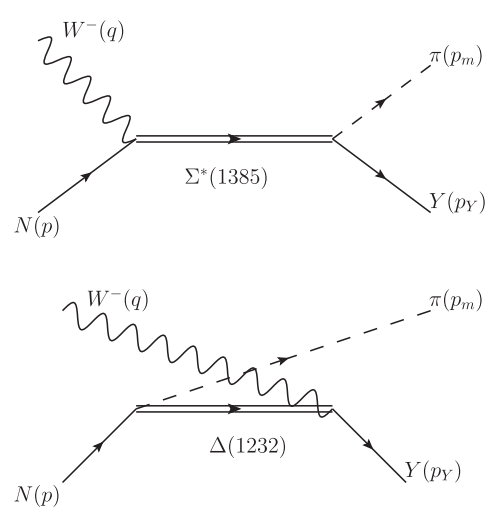

(b) Resonance diagrams included in our model. The s-channel $\Sigma^{*}(1385)$ diagram is shown in the upper figure, while the u-channel $\Delta(1232)$ diagram is depicted in the lower figure.

FIG. 1. Feynman diagrams included in our model for the Cabibbo suppressed $\pi Y$ production process off nucleons induced by antineutrinos. 


$$
\begin{aligned}
d^{9} \sigma= & \delta^{4}\left(p+q-p_{Y}-p_{m}\right) \frac{1}{(2 \pi)^{5} 4 M E_{\bar{\nu}}} \\
& \times \frac{d^{3} k^{\prime}}{2 E_{l}^{\prime}\left(\mathbf{k}^{\prime}\right)} \frac{d^{3} p_{m}}{2 E_{m}\left(\mathbf{p}_{m}\right)} \frac{d^{3} p_{Y}}{2 E_{Y}\left(\mathbf{p}_{Y}\right)} \sum \sum|\mathcal{M}|^{2},
\end{aligned}
$$

where the matrix element $\mathcal{M}$ is

$$
-i \mathcal{M}=-i \frac{G_{F}}{\sqrt{2}} \ell_{\mu} J_{H}^{\mu}
$$

with $G_{F}=\frac{\sqrt{2} g^{2}}{8 M_{W}^{2}}=1.1664 \times 10^{-5} \mathrm{GeV}^{-2}$ as the Fermi coupling constant and $\ell_{\mu}\left(J_{H}^{\mu}\right)$ is the lepton (hadron) current. For the final calculations, we use $J_{H}^{\mu}$ given as the sum of the hadronic currents of Eqs. (27)-(31) and (38)-(39). The symbol $\bar{\sum} \sum|\mathcal{M}|^{2}$ stands for the sum over final fermion spins and average over initial ones if these are, on average, unpolarized. In the present calculations, we take initial nucleons as unpolarized; however, antineutrinos are fully polarized, which leads to

$$
\bar{\sum} \sum|\mathcal{M}|^{2}=2 G_{F}^{2} L^{\mu \nu}\left(k, k^{\prime}\right) \sum_{\lambda_{N}, \lambda_{Y}} J_{\mu}^{H}\left(J_{\nu}^{H}\right)^{*}
$$

In the above expression, $L^{\mu \nu}\left(k, k^{\prime}\right)$ is the lepton tensor

$$
L^{\mu \nu}\left(k, k^{\prime}\right)=k^{\mu} k^{\prime \nu}+k^{\nu} k^{\prime \mu}-g^{\mu \nu}\left(k \cdot k^{\prime}\right)-i \epsilon^{\mu \nu \alpha \beta} k_{\alpha} k_{\beta}^{\prime},
$$

with $\epsilon^{0123}=1$. Finally, the sum over the spins of the initial and final baryons $\left(\lambda_{N, Y}\right)$ gives rise to traces over chains of Dirac matrices, of the form

$$
\begin{aligned}
W_{\mu \nu} & =\sum_{\lambda_{N}, \lambda_{Y}} J_{\mu}^{H}\left(J_{\nu}^{H}\right)^{*} \\
& =\sum_{\lambda_{N}, \lambda_{Y}}\left[\bar{u}_{\lambda_{Y}}\left(\mathbf{p}_{Y}\right) j_{\mu} u_{\lambda_{N}}(\mathbf{p})\right]\left[\bar{u}_{\lambda_{N}}(\mathbf{p}) \gamma^{0} j_{\nu}^{\dagger} \gamma^{0} u_{\lambda_{Y}}\left(\mathbf{p}_{Y}\right)\right] \\
& =\operatorname{Tr}\left[j_{\mu}(\not p+M) \gamma^{0} j_{\nu}^{\dagger} \gamma^{0}\left(\not \not Y_{Y}+M_{Y}\right)\right],
\end{aligned}
$$

where $j_{\mu}$ is the total hadron current $J_{\mu}^{H}$, but without Dirac spinors as given in Eqs. (27)-(31) and (38)-(39). For the calculation of Dirac traces, we have used the Mathematica package FEYNCALC [77-79].

The Eq. (2) can be further solved with the help of the $\delta$-function. The delta integration then fixes the cosine of the polar angle theta $\left(\theta_{m}^{0}=\cos ^{-1}\left[\hat{q} \cdot \hat{p}_{m}\right]\right)$ :

$$
\cos \theta_{m}^{0}=\frac{M_{Y}^{2}+\mathbf{q}^{2}+\mathbf{p}_{m}^{2}-\left(M+q^{0}-E_{m}\right)^{2}}{2|\mathbf{q}|\left|\mathbf{p}_{m}\right|},
$$

and the Eq. (2) thus reduces to,

$$
\begin{aligned}
d^{5} \sigma= & \frac{1}{(2 \pi)^{5} 4 M E_{\bar{\nu}}} \frac{\left|\mathbf{k}^{\prime}\right|}{8|\mathbf{q}|} \sum \sum|\mathcal{M}|^{2} \\
& \times \Theta\left(1-\cos ^{2} \theta_{m}^{0}\right) d E_{l}^{\prime} d \Omega_{\hat{k}^{\prime}} d E_{m} d \phi_{m},
\end{aligned}
$$

where $\phi_{m}$ is the azimuthal angle of the three-momentum of the $\pi$ meson on the reaction plane measured with respect to the $\bar{\nu}-l^{+}$scattering plane. The step function $(\Theta)$ puts a constraint on the cosine of theta $\left(\theta_{m}^{0}\right)$.

Finally, integrating Eq. (8) with respect to all the variables for a fixed antineutrino energy $E_{\bar{\nu}}$, we obtain

$$
\begin{aligned}
\sigma\left(E_{\bar{\nu}}\right)= & \frac{1}{(2 \pi)^{5} 4 M E_{\bar{\nu}}} \int d \Omega_{\hat{k}^{\prime}} \int_{m_{l}}^{E_{l \max }^{\prime}} d E_{l}^{\prime} \frac{\left|\mathbf{k}^{\prime}\right|}{8|\mathbf{q}|} \\
& \times \int_{m_{\pi}}^{E_{m}^{\max }} d E_{m} \Theta\left(1-\cos ^{2} \theta_{m}^{0}\right) \\
& \times \int_{0}^{2 \pi} d \phi_{m} \bar{\sum} \sum|\mathcal{M}|^{2} .
\end{aligned}
$$

For the upper limits of integration in the energies of the final lepton and the $\pi$ meson, we have chosen $E_{l \text { max }}^{\prime}=$ $E_{\bar{\nu}}+M-M_{Y}-m_{\pi}$ and $E_{m}^{\max }=E_{\bar{\nu}}-E_{l}^{\prime}+M-M_{Y}$.

\section{B. Born terms model}

Following Refs. [76,80] we can write the lowest order chiral Lagrangian in the SU(3) flavor scheme for mesons in the presence of an external weak charged current as

$$
\mathcal{L}_{M}^{(2)}=\frac{f_{\pi}^{2}}{4} \operatorname{Tr}\left[D_{\mu} U\left(D^{\mu} U\right)^{\dagger}\right]+\frac{f_{\pi}^{2}}{4} \operatorname{Tr}\left[\chi U^{\dagger}+U \chi^{\dagger}\right],
$$

where $f_{\pi}=93 \mathrm{MeV}$ is the pion decay constant, $U$ is the $\mathrm{SU}(3)$ representation of the pseudoscalar octet meson fields

$$
\begin{aligned}
& U(x)=\exp \left(i \frac{\phi(x)}{f_{\pi}}\right) \\
& \phi(x)=\left(\begin{array}{ccc}
\pi^{0}+\frac{\eta}{\sqrt{3}} & \sqrt{2} \pi^{+} & \sqrt{2} K^{+} \\
\sqrt{2} \pi^{-} & -\pi^{0}+\frac{\eta}{\sqrt{3}} & \sqrt{2} K^{0} \\
\sqrt{2} K^{-} & \sqrt{2} \bar{K}^{0} & -\frac{2}{\sqrt{3}} \eta
\end{array}\right) .
\end{aligned}
$$

$D_{\mu} U$ is the covariant derivative, given by

$$
D_{\mu} U=\partial_{\mu} U-i r_{\mu} U+i U l_{\mu},
$$

where $l_{\mu}$ and $r_{\mu}$ are left and right-handed external currents coupled to the meson fields. In the particular case of the weak charged current, these currents are

$$
r_{\mu}=0 \quad l_{\mu}=-\frac{g}{\sqrt{2}}\left(W_{\mu}^{+} T_{+}+W_{\mu}^{-} T_{-}\right),
$$


with $W_{\mu}^{ \pm}$the weak vector boson fields, $g$ the weak coupling constant, and $T_{ \pm}$the $3 \times 3$ matrices containing the Cabibbo-Kobayashi-Maskawa matrix elements relevant for the three flavor scheme,

$$
T_{+}=\left(\begin{array}{ccc}
0 & V_{u d} & V_{u s} \\
0 & 0 & 0 \\
0 & 0 & 0
\end{array}\right) ; \quad T_{-}=\left(\begin{array}{ccc}
0 & 0 & 0 \\
V_{u d} & 0 & 0 \\
V_{u s} & 0 & 0
\end{array}\right) .
$$

Finally, in Eq. (10), the symbol Tr denotes a trace over flavor space. The second term in Eq. (10) is not relevant for our study. It incorporates the explicit breaking of chiral symmetry due to the finite quark masses. With the Lagrangian given in Eq. (10) we can obtain the relevant $W K \pi$ and $W \bar{K}$ vertices necessary for the KP and KF diagrams shown in Fig. 1(a).

The lowest order interaction between the octet baryons, the octet meson and the weak external current can also be introduced following Ref. [76] as

$$
\begin{aligned}
\mathcal{L}_{M B}^{(1)}= & \operatorname{Tr}[\bar{B}(i \not D-M) B] \\
& +\frac{D}{2} \operatorname{Tr}\left[\bar{B} \gamma^{\mu} \gamma_{5}\left\{u_{\mu}, B\right\}\right]+\frac{F}{2} \operatorname{Tr}\left[\bar{B} \gamma^{\mu} \gamma_{5}\left[u_{\mu}, B\right]\right],
\end{aligned}
$$

where $B(x)$ is the $\mathrm{SU}(3)$ representation of the baryon fields

$$
B=\left(\begin{array}{ccc}
\frac{1}{\sqrt{2}} \Sigma^{0}+\frac{1}{\sqrt{6}} \Lambda & \Sigma^{+} & p \\
\Sigma^{-} & -\frac{1}{\sqrt{2}} \Sigma^{0}+\frac{1}{\sqrt{6}} \Lambda & n \\
\Xi^{-} & \Xi^{0} & -\frac{2}{\sqrt{6}} \Lambda
\end{array}\right) .
$$

The covariant derivative of the baryon fields is given in terms of the connection $\Gamma_{\mu}$ as

$$
D_{\mu} B=\partial_{\mu} B+\left[\Gamma_{\mu}, B\right]
$$

with

$$
\Gamma_{\mu}=\frac{1}{2}\left[u^{\dagger}\left(\partial_{\mu}-i r_{\mu}\right) u+u\left(\partial_{\mu}-i l_{\mu}\right) u^{\dagger}\right]
$$

In Eq. (18) we have introduced $u=\sqrt{U}=\exp \left(i \frac{\phi(x)}{2 f_{\pi}}\right)$. Also, in Eq. (15), the definition of the so-called vielbein, $u_{\mu}$, is given by

$$
u_{\mu}=i\left[u^{\dagger}\left(\partial_{\mu}-i r_{\mu}\right) u-u\left(\partial_{\mu}-i l_{\mu}\right) u^{\dagger}\right]
$$

In Eq. (15), $M$ represents the baryon mass matrix in the exact $\mathrm{SU}(3)$ limit with $D(=0.804)$ and $F(=0.463)$ as the symmetric and antisymmetric couplings, respectively. The two independent couplings appear because in the Clebsch-Gordan series expansion of two SU(3) octets $\{8\} \otimes\{8\}$, the $\{8\}$ representation is contained twice. These couplings can be measured from the baryon semileptonic decays within the Cabibbo model [81]. The Lagrangian of Eq. (15) allows to extract all the necessary vertices $N Y K, N Y K \pi, N Y W \pi$, and the leading order vector and axial-vector terms for the $N-Y$ strangeness-changing weak transitions for the diagrams depicted in Fig. 1(a). The latter can be written as

$$
\begin{gathered}
\left\langle Y\left(p_{Y}^{\prime}\right)\left|V^{\mu}\right| N(p)\right\rangle=\bar{u}_{Y}\left(\mathbf{p}_{Y}^{\prime}\right)\left[f_{1}^{N Y}\left(q^{2}\right) \gamma^{\mu}+i \frac{f_{2}^{N Y}\left(q^{2}\right)}{M+M_{Y}} \sigma^{\mu \nu} q_{\nu}+\frac{f_{3}^{N Y}\left(q^{2}\right)}{M+M_{Y}} q^{\mu}\right] u_{N}(\mathbf{p}) \\
\left\langle Y\left(p_{Y}^{\prime}\right)\left|A^{\mu}\right| N(p)\right\rangle=\bar{u}_{Y}\left(\mathbf{p}_{Y}^{\prime}\right)\left[g_{1}^{N Y}\left(q^{2}\right) \gamma^{\mu} \gamma_{5}+i \frac{g_{2}^{N Y}\left(q^{2}\right)}{M+M_{Y}} \sigma^{\mu \nu} \gamma_{5} q_{\nu}+\frac{g_{3}^{N Y}\left(q^{2}\right)}{M+M_{Y}} q^{\mu} \gamma_{5}\right] u_{N}(\mathbf{p}) .
\end{gathered}
$$

where $\left(g_{i}^{N Y}\right) f_{i}^{N Y}, i=1,2,3$ are the (axial-)vector form factors. The Lagrangian of Eq. (15) provides the values for the vector and axial couplings (form factors at $q^{2}=0$ ) $f_{1}^{N Y}(0)$ and $g_{1}^{N Y}(0)$, but not for the others, which may appear at higher orders of the chiral expansion. However, using symmetry arguments, one can get rid of some of them. For example, the weak electricity $\left[g_{2}^{N Y}\left(q^{2}\right)\right]$ and the scalar $\left[f_{3}^{N Y}\left(q^{2}\right)\right]$ form factors transform as second-class currents [82] under G-parity and are neglected for present calculations. ${ }^{1}$ In the present scheme the most standard way to obtain the $f_{2}(0)$ couplings is to include the relevant

\footnotetext{
${ }^{1} \mathrm{We}$ assume that G-parity is a good quantum number for the strong interactions and that in the Standard Model there are no second-class currents. Therefore, from here onward we neglect the contribution of $g_{2}$ and $f_{3}$. For an exhaustive discussion and implications of their effects in some observable if second-class currents are sizable, the reader is referred to Ref. [83] and references therein.
} 
pieces of the next higher order meson-baryon chiral Lagrangian [84] and to match the low energy constants to well-known $f_{2}(0)$ transition form factors, which can be obtained from Table I of Ref. [81].

Similar results could have been achieved by invoking exact $\mathrm{SU}(3)$ symmetry and the hypothesis that the weak vector currents and the electromagnetic one belong to the same octet of current operators of the $\mathrm{SU}(3)$ group. As the octet $\{8\}$ representation appears twice in the ClebschGordan series for the tensor product of two octets

\section{$\{8\} \otimes\{8\}$}

$$
=\{1\} \oplus\{8\} \oplus\left\{8^{\prime}\right\} \oplus\{10\} \oplus\{\overline{10}\} \oplus\{27\},
$$

this means that any octet operator connecting two octet baryons has two independent irreducible matrix elements. Therefore, it is necessary to explicitly calculate two independent matrix elements for an octet operator. Later, using the SU(3) Wigner-Eckart theorem, all the nonvanishing matrix elements between octet states connected through an octet current operator can be related through the SU(3) Clebsch-Gordan coefficients, which can be found in Ref. [85], with the previous explicitly calculated two matrix elements. In the case of the octet of vector currents, these two irreducible matrix elements can be written in terms of the proton and neutron electromagnetic current matrix elements, $\left\langle p\left|J_{\mathrm{em}}^{\mu}\right| p\right\rangle$ and $\left\langle n\left|J_{\mathrm{em}}^{\mu}\right| n\right\rangle$. This facilitates us to express all the $N \rightleftharpoons Y$ transition vector form factors in terms of those, $f_{1,2}^{p, n}\left(q^{2}\right)$, of the electromagnetic interaction, that is well measured. They are summarized in Table I, and for present work we use the Galster parametrization [86] for the electromagnetic form factors.

A similar argument may be given for the axial-vector currents in the Cabibbo model. However, in this case, there are not two well-measured independent transition matrix elements to be used to define univocally the rest of the transition matrix elements driven by the weak axial current.
The only known parameter we have is for the $n \rightarrow p$ weak transition, from where one can extract the axial coupling of the nucleon, $g_{A}(0)=g_{1}^{n p}(0)=1.267$. Normally, its $q^{2}$-dependence is assumed to have a dipole form with an axial mass of $M_{A}=1.03 \mathrm{GeV}$,

$$
g_{A}\left(q^{2}\right)=\frac{g_{A}(0)}{\left(1-\frac{q^{2}}{M_{A}^{2}}\right)^{2}},
$$

where $g_{A}(0)=D+F$. One assumption that has been extensively used in past works $[67,70,75,83,87]$ assumes that the $q^{2}$-dependence acquired by the $D$ and $F$ couplings is identical and driven by the dependence on $q^{2}$ of the nucleon axial form factor $g_{A}\left(q^{2}\right)$. Under this assumption we can write

$$
\begin{aligned}
g_{1}^{N Y}\left(q^{2}\right) & =a D_{A}\left(q^{2}\right)+b F_{A}\left(q^{2}\right)=\frac{a D+b F}{\left(1-\frac{q^{2}}{M_{A}^{2}}\right)^{2}} \\
& =\frac{a D+b F}{D+F} \frac{g_{A}(0)}{\left(1-\frac{q^{2}}{M_{A}^{2}}\right)^{2}}=\frac{a D+b F}{D+F} g_{A}\left(q^{2}\right),
\end{aligned}
$$

where $a$ and $b$ are SU(3) Clebsch-Gordan coefficients, and $D_{A}$ and $F_{A}$ are normalized to $D$ and $F$ couplings at $q^{2}=0$. The values for these axial-vector form factors are tabulated in Table II for the transitions of interest for our work.

Finally, invoking partial conservation of the axial current (PCAC) in the chiral limit, we can relate the induced pseudoscalar $g_{3}^{N Y}\left(q^{2}\right)$ form factor with the axial one, $g_{1}^{N Y}\left(q^{2}\right)$ :

$$
g_{3}^{N Y}\left(q^{2}\right)=-g_{1}^{N Y}\left(q^{2}\right) \frac{\left(M+M_{Y}\right)^{2}}{q^{2}}
$$

Finally, to take into account the nonvanishing meson masses, the denominator is extrapolated from $q^{2}$ to a kaon

TABLE I. Dirac and Pauli vector form factors for the weak strangeness-changing transitions considered in this work.

\begin{tabular}{lccc}
\hline \hline$i=1,2$ & $Y=\Lambda$ & $Y=\Sigma^{0}$ & $Y=\Sigma^{-}$ \\
\hline$f_{i}^{p Y}\left(q^{2}\right)$ & $-\sqrt{\frac{3}{2}} f_{i}^{p}\left(q^{2}\right)$ & $-\frac{1}{\sqrt{2}}\left(f_{i}^{p}\left(q^{2}\right)+2 f_{i}^{n}\left(q^{2}\right)\right)$ & 0 \\
$f_{i}^{n Y}\left(q^{2}\right)$ & 0 & 0 & $-\left(f_{i}^{p}\left(q^{2}\right)+2 f_{i}^{n}\left(q^{2}\right)\right)$ \\
\hline \hline
\end{tabular}

TABLE II. $\quad g_{1}^{N Y}\left(q^{2}\right)$ axial-vector form factors for the weak strangeness-changing transitions considered in this work. The definition of $x=\frac{F}{D+F}$ is taken for simplicity in the formulae.

\begin{tabular}{lccc}
\hline \hline & $Y=\Lambda$ & $Y=\Sigma^{0}$ & $Y=\Sigma^{-}$ \\
\hline$g_{1}^{p Y}\left(q^{2}\right)$ & $-\sqrt{\frac{1}{6}}(1+2 x) g_{A}\left(q^{2}\right)$ & $\frac{1}{\sqrt{2}}(1-2 x) g_{A}\left(q^{2}\right)$ & 0 \\
$g_{1}^{n Y}\left(q^{2}\right)$ & 0 & 0 & $(1-2 x) g_{A}\left(q^{2}\right)$ \\
\hline \hline
\end{tabular}


pole, $q^{2}-M_{K}^{2}$, for strangeness-changing axial weak charged currents. This is called the kaon-pole dominance [88], and it is equivalent to assume that the induced pseudoscalar form factor is generated through the coupling of the $W^{-}$boson to the baryons through a $K^{-}$, as depicted in Fig. 2. Although the kaon-pole dominance is expected to work worse than the pion-pole dominance for nonstrangeness-changing weak axial currents, the contribution of the pseudoscalar form factor $g_{3}^{N Y}\left(q^{2}\right)$ is proportional to $q^{\mu}$ and hence to the lepton mass; therefore, its contribution would be too small for muon and electron antineutrinos induced reactions.

While deriving Eq. (25), the baryons in Fig. 2 are taken as on-shell. The off-shellness of intermediate baryons can be restored by replacing the $\left(M+M_{Y}\right)$ in the numerator with an operator that reduces this factor when both baryons are on-shell. That can easily be achieved by substituting the axial vertex of Eq. (21) by

$$
\begin{aligned}
& \left\langle Y\left(p_{Y}^{\prime}\right)\left|A^{\mu}\right| N(p)\right\rangle \\
& \quad=g_{1}^{N Y}\left(q^{2}\right) \bar{u}_{Y}\left(\mathbf{p}_{Y}^{\prime}\right)\left(\gamma^{\mu} \gamma_{5}-\frac{q^{\mu} \mathscr{q}}{q^{2}-M_{K}^{2}} \gamma_{5}\right) u_{N}(\mathbf{p}),
\end{aligned}
$$

where we used the relationship,

$$
\bar{u}_{Y}\left(\mathbf{p}_{Y}^{\prime}\right) q \gamma_{5} u_{N}(\mathbf{p})=\left(M+M_{Y}\right) \bar{u}_{Y}\left(\mathbf{p}_{Y}^{\prime}\right) \gamma_{5} u_{N}(\mathbf{p})
$$

when both baryons are on-shell.

Now, applying the Feynman rules to the vertices and propagators appearing in Fig. 1(a), which can be extracted from the Lagrangians given in Eqs. (10) and (15), we obtain the following hadron currents for the Born term diagrams:

$$
J_{\mathrm{CT}}^{\mu}=i V_{u s} \mathcal{A}_{\mathrm{CT}}^{N \rightarrow Y \pi} F_{D}\left(q^{2}\right) \bar{u}_{Y}\left(\mathbf{p}_{Y}\right)\left[\gamma^{\mu}-a^{N \rightarrow Y \pi} \gamma^{\mu} \gamma_{5}\right] u_{N}(\mathbf{p})
$$

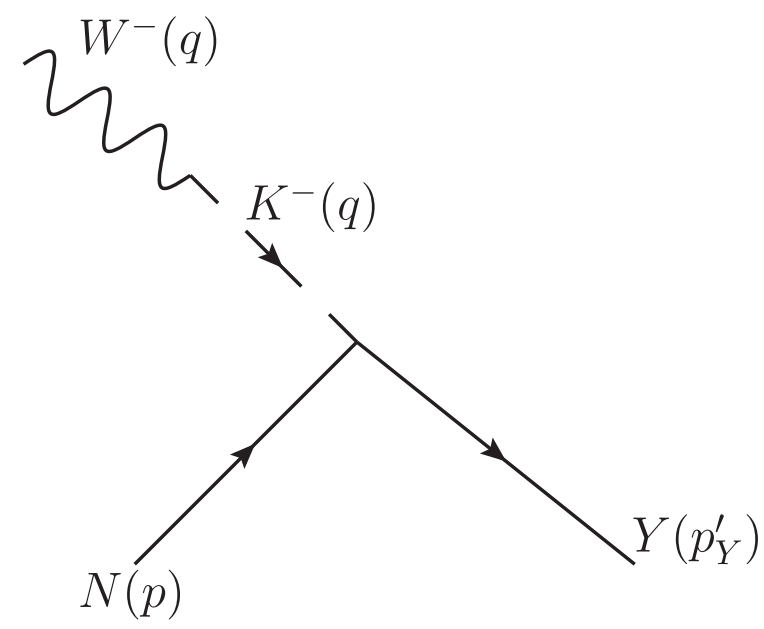

FIG. 2. Feynman diagram illustrating the generation of the pseudoscalar term in the axial-vector current.

$$
\begin{aligned}
J_{\mathrm{KP}}^{\mu}= & i V_{u s} \mathcal{A}_{\mathrm{KP}}^{N \rightarrow Y \pi} F_{D}\left(q^{2}\right) \frac{q^{\mu}}{q^{2}-M_{K}^{2}} \bar{u}_{Y}\left(\mathbf{p}_{Y}\right) \\
& \times\left[q-\frac{\left(M_{Y}-M\right)}{2}\right] u_{N}(\mathbf{p}) \\
J_{\mathrm{KF}}^{\mu}= & i V_{u s} \mathcal{A}_{\mathrm{KF}}^{N \rightarrow Y \pi} F_{D}\left(q^{2}\right) \frac{2 p_{m}^{\mu}-q^{\mu}}{\left(p_{m}-q\right)^{2}-M_{K}^{2}}\left(M_{Y}+M\right) \\
\times & \bar{u}_{Y}\left(\mathbf{p}_{Y}\right) \gamma_{5} u_{N}(\mathbf{p}) \\
J_{\mathrm{s}-\mathrm{Y}^{\prime}}^{\mu}= & i V_{u s} \mathcal{A}_{\mathrm{s}-\mathrm{Y}^{\prime}}^{N \rightarrow Y \pi} \bar{u}_{Y}\left(\mathbf{p}_{Y}\right) \not p_{m} \gamma_{5} \frac{\not p+q 1+M_{Y^{\prime}}}{(p+q)^{2}-M_{Y^{\prime}}^{2}} \\
& \times\left[V_{N Y^{\prime}}^{\mu}(q)-A_{N Y^{\prime}}^{\mu}(q)\right] u_{N}(\mathbf{p}) \\
J_{\mathrm{u}-\mathrm{N}^{\prime}}^{\mu}= & i V_{u s} \mathcal{A}_{\mathrm{u}-\mathrm{N}^{\prime}}^{N \rightarrow Y \pi} \bar{u}_{Y}\left(\mathbf{p}_{Y}\right)\left[V_{N^{\prime} Y}^{\mu}(q)-A_{N^{\prime} Y}^{\mu}(q)\right] \\
& \times \frac{\not p-\not p_{m}+M}{\left(p-p_{m}\right)^{2}-M^{2}} \not \not_{m} \gamma_{5} u_{N}(\mathbf{p}),
\end{aligned}
$$

where $Y, Y^{\prime}=\Sigma, \Lambda ; N, N^{\prime}=p, n ; F_{D}\left(q^{2}\right)$ is a global dipole form factor ${ }^{2}$

$$
F_{D}\left(q^{2}\right)=\frac{1}{\left(1-\frac{q^{2}}{M_{D}^{2}}\right)^{2}}, \quad M_{D} \simeq 1 \mathrm{GeV} .
$$

for the CT, KP and KF diagrams. In Eqs. (27)-(31), the $\mathcal{A}_{i}^{N \rightarrow Y \pi}$ are global constants that depend on the particular reaction given in Table III.

Finally, the vector and axial-vector weak vertices of Eqs. (30) and (31) are given by

$$
\begin{aligned}
& V_{N Y^{\prime}}^{\mu}(q)=f_{1}^{N Y^{\prime}}\left(q^{2}\right) \gamma^{\mu}+\frac{i f_{2}^{N Y^{\prime}}\left(q^{2}\right)}{M+M_{Y^{\prime}}} \sigma^{\mu \nu} q_{\nu} \\
& A_{N Y^{\prime}}^{\mu}(q)=g_{1}^{N Y^{\prime}}\left(q^{2}\right)\left(\gamma^{\mu}-\frac{q^{\mu} q^{2}}{q^{2}-M_{K}^{2}}\right) \gamma_{5},
\end{aligned}
$$

with the vector $f_{1,2}^{N Y^{\prime}}\left(q^{2}\right)$ and axial-vector $g_{1}^{N Y^{\prime}}\left(q^{2}\right)$ form factors given in Tables I and II, respectively.

The fact that the CT and KP diagrams for the $p \rightarrow \Sigma^{-} \pi^{+}$ channel are zero and not for the other ones can be explained with the help of Figs. 3(a) and 3(b). The key is not to need to emit gluons in these diagrams, i.e., that the virtual $s \bar{u}$ pair $\left(K^{-}\right)$in which the $W^{-}$decays could be redistributed along with the quarks of the initial nucleon in the two final hadrons, the hyperon and the pion, but without the need of emitting gluons to create a $q \bar{q}$ pair of the same flavor. It seems to be a kind of OZI forbidding rule because the valence quarks of the initial $W^{-} N$ state get fully redistributed into the final $Y \pi$ state without any gluon emission. This is totally possible for all the channels except for the $W^{-} p \rightarrow \Sigma^{-} \pi^{+}$as shown in Fig. 3(b). Notice that the $\bar{u}$

\footnotetext{
${ }^{2}$ This same assumption for this global dipole form factor has also been taken in previous works such as those of Refs. [62,72-74].
} 
TABLE III. Constants $\mathcal{A}_{i}^{N \rightarrow Y \pi}$ and $a^{N \rightarrow Y \pi}$ (for the axial-vector piece of the CT diagram) for each reaction and diagram in our model.

\begin{tabular}{lccccccc}
\hline \hline Reaction & $\mathcal{A}_{\mathrm{CT}}^{N \rightarrow Y \pi}$ & $a^{N \rightarrow Y \pi}$ & $\mathcal{A}_{\mathrm{KP}}^{N \rightarrow Y \pi}$ & $\mathcal{A}_{\mathrm{KF}}^{N \rightarrow Y \pi}$ & $\mathcal{A}_{\mathrm{s}-\Sigma}^{N \rightarrow Y \pi}$ & $\mathcal{A}_{\mathrm{u}-\mathrm{N}^{\prime}}^{N \rightarrow \pi}$ & $\mathcal{A}_{\mathrm{s}-\Lambda}^{N \rightarrow Y \pi}$ \\
\hline $\bar{\nu}_{l}+p \rightarrow l^{+}+\pi^{0}+\Lambda$ & $\frac{\sqrt{3}}{2 \sqrt{2} f_{\pi}}$ & $F+\frac{D}{3}$ & $-\frac{\sqrt{3}}{2 \sqrt{2} f_{\pi}}$ & $-\frac{(D+3 F)}{2 \sqrt{6} f_{\pi}}$ & $\frac{D}{\sqrt{3} f_{\pi}}$ & $\frac{D+F}{2 f_{\pi}}$ & 0 \\
$\bar{\nu}_{l}+n \rightarrow l^{+}+\pi^{-}+\Lambda$ & $\frac{\sqrt{3}}{2 f_{\pi}}$ & $F+\frac{D}{3}$ & $-\frac{\sqrt{3}}{2 f_{\pi}}$ & $-\frac{(D+3 F)}{2 \sqrt{3} \pi_{\pi}}$ & $\frac{D}{\sqrt{3} f_{\pi}}$ & $\frac{D+F}{\sqrt{2} f_{\pi}}$ & 0 \\
$\bar{\nu}_{l}+p \rightarrow l^{+}+\pi^{0}+\Sigma^{0}$ & $\frac{1}{2 \sqrt{2} f_{\pi}}$ & $F-D$ & $-\frac{1}{2 \sqrt{2} f_{\pi}}$ & $\frac{(D-F)}{2 \sqrt{2} f_{\pi}}$ & 0 & $\frac{D+F}{2 f_{\pi}}$ & $\frac{D}{\sqrt{3} f_{\pi}}$ \\
$\bar{\nu}_{l}+p \rightarrow l^{+}+\pi^{-}+\Sigma^{+}$ & $\frac{1}{\sqrt{2} f_{\pi}}$ & $F-D$ & $-\frac{1}{\sqrt{2} f_{\pi}}$ & $\frac{(D-F)}{\sqrt{2} f_{\pi}}$ & $-\frac{F}{f_{\pi}}$ & 0 & $\frac{D}{\sqrt{3} f_{\pi}}$ \\
$\bar{\nu}_{l}+p \rightarrow l^{+}+\pi^{+}+\Sigma^{-}$ & 0 & 0 & 0 & 0 & $\frac{F}{f_{\pi}}$ & $\frac{D+F}{\sqrt{2} f_{\pi}}$ & $\frac{D}{\sqrt{3} f_{\pi}}$ \\
$\bar{\nu}_{l}+n \rightarrow l^{+}+\pi^{-}+\Sigma^{0}$ & $-\frac{1}{2 f_{\pi}}$ & $F-D$ & $\frac{1}{2 f_{\pi}}$ & $\frac{(F-D)}{2 f_{\pi}}$ & $\frac{F}{f_{\pi}}$ & $\frac{D+F}{\sqrt{2} f_{\pi}}$ & 0 \\
$\bar{\nu}_{l}+n \rightarrow l^{+}+\pi^{0}+\Sigma^{-}$ & $\frac{1}{2 f_{\pi}}$ & $F-D$ & $-\frac{1}{2 f_{\pi}}$ & $\frac{(D-F)}{2 f_{\pi}}$ & $-\frac{F}{f_{\pi}}$ & $-\frac{D+F}{2 f_{\pi}}$ & 0 \\
\hline \hline
\end{tabular}

antiquark coming out from the decay of the $W^{-}$is not present in the final state. Therefore, it is completely necessary to annihilate it with an $u$ quark via gluon emission to have the right quarks in the final state.

As the $s \bar{u}$ quark-antiquark pair has the same quantum numbers as the $K^{-}$, this argument holds not only for the CT diagram, but for the KP as well.

It is worth noting that these findings are in agreement for the tree level amplitudes for the reaction channel $p \rightarrow$ $\Sigma^{-} \pi^{+}$with those of Ref. [62], where the authors consider the CT, KP and KF reaction mechanisms.

This argument also explains why in Ref. [75] there were no $\mathrm{CT}$ and KP amplitudes at tree level.

\section{Resonance model}

To describe the currents of the resonance diagrams depicted in Fig. 1(b), we follow the prescription discussed in Refs. [27,34,73-75] and include the lowest lying resonances belonging to the decuplet representation of the SU(3) group. The resonant states which may appear in the s-channel and u-channel are $\Sigma^{*}(1385)$ and $\Delta(1232)$, respectively.

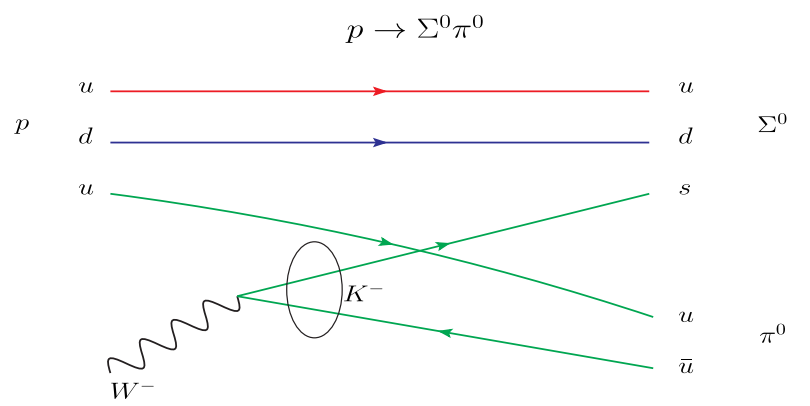

(a) In this diagram for the channel $p \rightarrow \Sigma^{0} \pi^{0}$, the valence quarks of the initial state particles can be fully accommodated

in the final state particles without any gluon emission.
Though the $\Delta(1232)$ resonances are widely studied in the literature, there is less information available for the $\Sigma^{*}(1385)$ resonances. However, we know that both $\Sigma^{*}(1385)$ and $\Delta(1232)$ are members of the same decuplet, therefore under the assumption of exact $\mathrm{SU}(3)$ flavor symmetry for the couplings and using the Eq. (22), the weak transition form factors connecting an octet state to a decuplet state can be obtained. One should notice that as the weak charged current belongs to the octet representation of current operators of the SU(3) group, and couples one octet state with a decuplet state, the representation $\{10\}$ appears only once in the Clebsch-Gordan series of Eq. (22). Therefore, there is only one independent reduced matrix element. We will take for the latter the transition matrix element as:

$$
\left\langle\Delta^{+}\left(p_{R}\right)\left|j_{\Delta S=0}^{\mu}\right| n(p)\right\rangle=\bar{u}_{\alpha}\left(\mathbf{p}_{R}\right) \Gamma^{\alpha \mu}(p, q) u(\mathbf{p}),
$$

with $p_{R}=p+q$. In Eq. (33), $\Gamma^{\alpha \mu}(p, q)$ is the vertex function given by

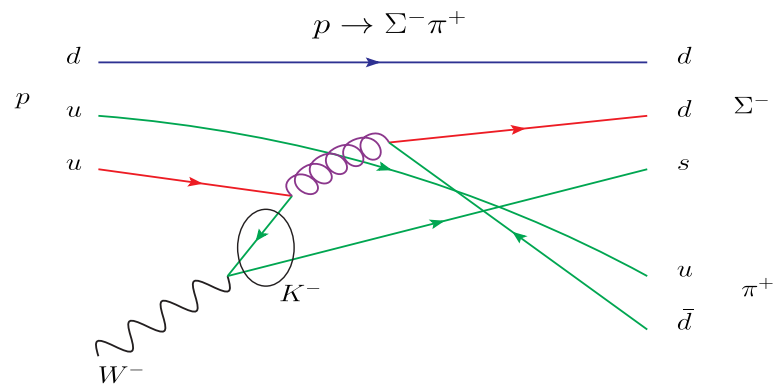

(b) In this diagram for the channel $p \rightarrow \Sigma^{-} \pi^{+}$, all the valence quarks of the initial state particles cannot be fully accommodated in the final state particles without gluon emission and the creation of a $d \bar{d}$ pair.

FIG. 3. Two possible Feynman diagrams in terms of quarks and gluons to explain why the CT and KP diagrams are forbidden for the $p \rightarrow \Sigma^{-} \pi^{+}$reaction channel but not for the others. The colored quark lines represent their possible colors in QCD to make colorless initial and final hadrons. 


$$
\begin{aligned}
\Gamma^{\alpha \mu}(p, q)= & {\left[\frac{C_{3}^{V}}{M}\left(g^{\alpha \mu} q-q^{\alpha} \gamma^{\mu}\right)+\frac{C_{4}^{V}}{M^{2}}\left(g^{\alpha \mu} q \cdot(p+q)-q^{\alpha}(p+q)^{\mu}\right)+\frac{C_{5}^{V}}{M^{2}}\left(g^{\alpha \mu} q \cdot p-q^{\alpha} p^{\mu}\right)+C_{6}^{V} g^{\alpha \mu}\right] \gamma_{5} } \\
& +\left[\frac{C_{3}^{A}}{M}\left(g^{\alpha \mu} q-q^{\alpha} \gamma^{\mu}\right)+\frac{C_{4}^{A}}{M^{2}}\left(g^{\alpha \mu} q \cdot(p+q)-q^{\alpha}(p+q)^{\mu}\right)+C_{5}^{A} g^{\alpha \mu}+\frac{C_{6}^{A}}{M^{2}} q^{\alpha} q^{\mu}\right]
\end{aligned}
$$

$\bar{u}_{\alpha}\left(\mathbf{p}_{R}\right)$ is a Rarita-Schwinger spinor describing spin- $\frac{3}{2}$ particles, and $j_{\Delta S=0}^{\mu}$ is the strangeness-preserving weak charged current coupled to an incoming $W^{+}$boson.

A systematic way of obtaining the relationships (SU(3) factors) between the weak vertices for all the allowed transitions and that for the $n \rightarrow \Delta^{+}$[given in Eq. (34)] is to use the lowest order Lagrangian that couples the decuplet baryons with the octet baryons and mesons in the presence of an external current $[89,90]$ and that was already used in Refs. [73-75]. Its form is

$$
\mathcal{L}_{\mathrm{dec}}=\mathcal{C}\left(\epsilon^{a b c} \bar{T}_{a d e}^{\mu}\left(u_{\mu}\right)_{b}^{d} B_{c}^{e}+\epsilon^{a b c} \bar{B}_{e}^{c}\left(u_{\mu}\right)_{d}^{b} T_{a e d}^{\mu}\right)
$$

where $B$ is given by Eq. (16), $u_{\mu}$ is the vielbein of Eq. (19), and $T_{\text {aed }}^{\mu}$ is the $\mathrm{SU}(3)$ representation of the RaritaSchwinger fields for the decuplet baryons. This representation is completely symmetric in the three flavor indices, and an implicit sum over flavor indices $(a, b, \ldots=1,2,3)$ is understood in Eq. (35). It is worth relating the $T_{a b c}$ representation to the physical states ${ }^{3}$ :

$$
\begin{array}{lrlrl}
T_{111}=\Delta^{++} ; & T_{112}=\frac{\Delta^{+}}{\sqrt{3}} ; & T_{122}=\frac{\Delta^{0}}{\sqrt{3}} \\
T_{222}=\Delta^{-} ; & T_{113}=\frac{\Sigma^{*+}}{\sqrt{3}} ; & T_{123}=\frac{\Sigma^{* 0}}{\sqrt{6}} \\
T_{223}=\frac{\Sigma^{*-}}{\sqrt{3}} ; & T_{133}=\frac{\Xi^{* 0}}{\sqrt{3}} ; & T_{233}=\frac{\Xi^{*-}}{\sqrt{3}} \\
T_{333}=\Omega^{-} . & &
\end{array}
$$

The Lagrangian of Eq. (35) only provides the leading weak axial coupling $C_{5}^{A}(0)$ for all the allowed weak transitions. Knowing that $\left.C_{5}^{A}(0)\right|_{n \rightarrow \Delta^{+}} \simeq \frac{2 \mathcal{C}}{\sqrt{3}}$ with $\mathcal{C} \sim 1$ $[73,75]$, we can relate all the other leading axial couplings for the other weak transitions to the $n \rightarrow \Delta^{+}$one. These relative factors are then applied to all the vector $C_{i}^{V}\left(q^{2}\right)$ and axial $C_{i}^{A}\left(q^{2}\right)$ form factors, thus assuming exact $\mathrm{SU}(3)$ symmetry for the couplings. ${ }^{4}$ We choose the form factors for the $n \rightarrow \Delta^{+}$transition given in Ref. [27] with the exception that for strangeness-changing processes

\footnotetext{
${ }^{3}$ Note that there is a typographical mistake in $T_{233}$ for the $\Xi^{*-}$ state in the footnotes of Refs. [73,74].

${ }^{4}$ In Appendix, we give an equivalent formulation based on flavor SU(3) symmetry.
}

$$
C_{6}^{A}\left(q^{2}\right)=C_{5}^{A}\left(q^{2}\right) \frac{M^{2}}{M_{K}^{2}-q^{2}}
$$

which appears when one imposes PCAC for the transition similar to Fig. 2 with the final hyperon replaced by the $\Sigma^{*}(1385)$ resonance. Note that the strong coupling $\mathcal{C} \simeq 1$ is obtained to match the $\Delta$ width at its nominal mass. If we apply the Feynman rules to the diagrams depicted in Fig. 1(b), we obtain the following amplitudes:

$$
\begin{aligned}
J_{\mathrm{s}-\Sigma^{*}}^{\mu}= & i V_{u s} \mathcal{A}_{\mathrm{s}-\Sigma^{*}}^{N \rightarrow} \frac{p_{m}^{\beta}}{p_{\Sigma^{*}}^{2}-M_{\Sigma^{*}}^{2}+i M_{\Sigma^{*}} \Gamma_{\Sigma^{*}}} \bar{u}_{Y}\left(\mathbf{p}_{Y}\right) \mathcal{P}_{\beta \alpha}\left(p_{\Sigma^{*}}\right) \\
& \times \Gamma^{\alpha \mu}(p, q) u_{N}(\mathbf{p})
\end{aligned}
$$

$$
\begin{aligned}
J_{\mathrm{u}-\Delta}^{\mu}= & i V_{u s} \mathcal{A}_{\mathrm{u}-\Delta}^{N \rightarrow Y \pi} \frac{p_{m}^{\beta}}{p_{\Delta}^{2}-M_{\Delta}^{2}+i M_{\Delta} \Gamma_{\Delta}} \bar{u}_{Y}\left(\mathbf{p}_{Y}\right) \tilde{\Gamma}^{\mu \alpha}\left(p_{Y}, q\right) \\
& \times \mathcal{P}_{\alpha \beta}\left(p_{\Delta}\right) u_{N}(\mathbf{p}),
\end{aligned}
$$

where $\quad p_{\Sigma^{*}}=p+q, \quad p_{\Delta}=p-p_{m}, \quad \tilde{\Gamma}^{\mu \alpha}\left(p_{Y}, q\right)=$ $\gamma^{0}\left[\Gamma^{\alpha \mu}\left(p_{Y},-q\right)\right]^{\dagger} \gamma^{0}, \mathcal{P}_{\alpha \beta}\left(p_{D}\right)$ is the spin- $\frac{3}{2}$ projector operator appearing in the propagator of Rarita-Schwinger fields, and given by

$$
\begin{aligned}
\mathcal{P}_{\alpha \beta}(P)= & -\left(\not P+M_{D}\right)\left[g_{\alpha \beta}-\frac{1}{3} \gamma_{\alpha} \gamma_{\beta}\right. \\
& \left.-\frac{2}{3} \frac{P_{\alpha} P_{\beta}}{M_{D}^{2}}+\frac{1}{3} \frac{P_{\alpha} \gamma_{\beta}-P_{\beta} \gamma_{\alpha}}{M_{D}}\right],
\end{aligned}
$$

with $M_{D}$ the corresponding mass of the decuplet baryon, either the $\Delta$ or the $\Sigma^{*}$, and $P$ the four-momentum carried by

TABLE IV. Constants $\mathcal{A}_{i}^{N \rightarrow Y \pi}$ for each reaction and the resonances $\left(\mathrm{s}-\Sigma^{*}\right.$ and $\left.\mathrm{u}-\Delta\right)$ diagrams of Fig. 1(b) in our model.

\begin{tabular}{lcc}
\hline \hline Reaction & $\mathcal{A}_{\mathrm{s}-\Sigma^{*}}^{N \rightarrow Y_{n}}$ & $\mathcal{A}_{\mathrm{u}-\Delta}^{N \rightarrow Y \pi}$ \\
\hline $\bar{\nu}_{l}+p \rightarrow l^{+}+\pi^{0}+\Lambda$ & $\frac{\mathcal{C}}{\sqrt{2} f_{\pi}}$ & 0 \\
$\bar{\nu}_{l}+n \rightarrow l^{+}+\pi^{-}+\Lambda$ & $\frac{\mathcal{C}}{f_{\pi}}$ & 0 \\
$\bar{\nu}_{l}+p \rightarrow l^{+}+\pi^{0}+\Sigma^{0}$ & 0 & $2 \sqrt{\frac{2}{3} \frac{\mathcal{C}}{f_{\pi}}}$ \\
$\bar{\nu}_{l}+p \rightarrow l^{+}+\pi^{-}+\Sigma^{+}$ & $\frac{\mathcal{C}}{\sqrt{6} f_{f_{\pi}}}$ & $\frac{\mathcal{C} \sqrt{6}}{f_{\pi}}$ \\
$\bar{\nu}_{l}+p \rightarrow l^{+}+\pi^{+}+\Sigma^{-}$ & $-\frac{\mathcal{C}}{\sqrt{6} f_{\pi}}$ & $\sqrt{\frac{2}{3}} \frac{\mathcal{C}}{f_{\pi}}$ \\
$\bar{\nu}_{l}+n \rightarrow l^{+}+\pi^{-}+\Sigma^{0}$ & $-\frac{\mathcal{C}}{\sqrt{3} f_{\pi}}$ & $-\frac{2 \mathcal{C}}{\sqrt{3} f_{\pi}}$ \\
$\bar{\nu}_{l}+n \rightarrow l^{+}+\pi^{0}+\Sigma^{-}$ & $\frac{\mathcal{C}}{\sqrt{3} f_{\pi}}$ & $\frac{2 \mathcal{V}}{\sqrt{3} f_{\pi}}$ \\
\hline \hline
\end{tabular}



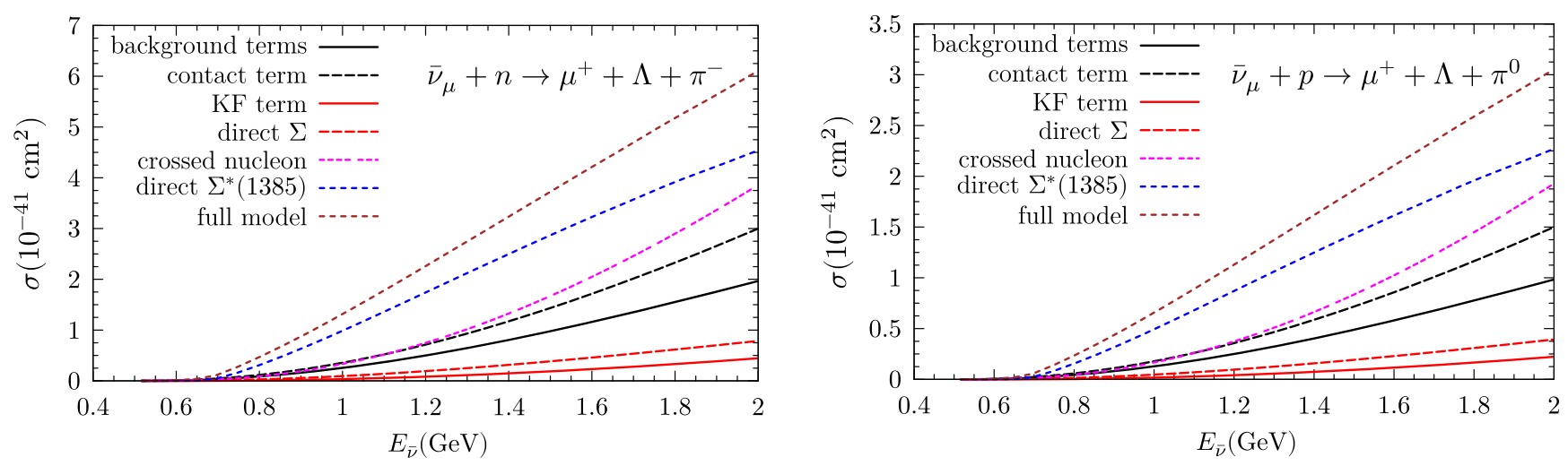

FIG. 4. Total cross sections for the $\Lambda$ hyperon production off neutrons (left panel) and protons (right panel). Some of the contributions of individual diagrams of Figs. 1(a) and 1(b) have been singled out. Note that the nature is identical in both panels, except the scale in the vertical axis. This is because the total cross section for neutrons is exactly twice that for protons (see Appendix).

these particles. The constants $\mathcal{A}_{i}^{N \rightarrow Y \pi}$ appearing in Eqs. (38) and (39) are given in Table IV.

Finally, in Eq. (38), $\Gamma_{\Sigma^{*}}$ is the energy dependent $\Sigma^{*}(1385)$ width, given by

$$
\Gamma_{\Sigma^{*}}=\Gamma_{\Lambda \pi}+\Gamma_{\Sigma \pi}+\Gamma_{N \bar{K}}+\Gamma_{\Sigma \eta}+\Gamma_{\Xi K},
$$

where the different strong partial widths $\Gamma_{B \phi}$ can be calculated with the vertices from the Lagrangian given in Eq. (35). Their expressions are always the same up to an $\mathrm{SU}(3)$ factor and are given as

$$
\begin{aligned}
\Gamma_{D \rightarrow B \phi}= & \frac{C_{B \phi}}{192 \pi}\left(\frac{\mathcal{C}}{f_{\pi}}\right)^{2} \frac{\left(W+M_{B}\right)^{2}-m_{\phi}^{2}}{W^{5}} \\
& \times \lambda^{3 / 2}\left(W^{2}, M_{B}^{2}, m_{\phi}^{2}\right) \Theta\left(W-M_{B}-m_{\phi}\right),
\end{aligned}
$$

where $\lambda(x, y, z)=x^{2}+y^{2}+z^{2}-2 x y-2 x z-2 y z$ is the Källen $\lambda$-function, $M_{B}$ and $m_{\phi}$ are the final baryon and meson masses in the decay of the $\Sigma^{*}, \Theta$ is the unit step function allowing a partial width to decay into channels $B \phi$, only when the invariant mass squared $W^{2}=(p+q)^{2}$ carried by the resonance is higher than the threshold $\left(M_{B}+m_{\phi}\right)^{2}$. Finally, the $\mathrm{SU}(3)$ factors $C_{B \phi}$ are 1 for $\Lambda \pi$ and $\Sigma \eta$, while they are $\frac{2}{3}$ for the $\Sigma \pi, N \bar{K}$ and $\Xi K$.

In Eq. (39), for the $\Delta(1232)$ appearing in u-channel diagram we take $\Gamma_{\Delta} \rightarrow 0$ for the present kinematics, as the four momentum $p_{\Delta}\left(=p-p_{m}\right)$ squared is always below the decay threshold. Indeed,

$$
\begin{aligned}
p_{\Delta}^{2} & =M^{2}+m_{\pi}^{2}-2 M E_{\pi} \leqslant\left(M-m_{\pi}\right)^{2}<\left(M+m_{\pi}\right)^{2} \\
& <M_{\Delta}^{2} .
\end{aligned}
$$

This leads to the $\Delta$ width equals zero as $p_{\Delta}^{2}<\left(M+m_{\pi}\right)^{2}$ holds for all the kinematics regions under consideration.

\section{RESULTS}

\section{A. Total cross sections}

In Figs. 4-6 we show results for the total cross sections off proton and neutron targets, as a function of the muonantineutrino energy in the LAB frame. In order to understand the dynamics of the reaction channels, we show the contribution due to individual diagrams of Figs 1(a) and 1(b), wherever applicable. One may notice that we do not give the results for all the channels due to the KP diagram of Fig. 1(a). This is because the hadron tensor associated with the KP diagram alone is proportional to $q^{\mu} q^{\nu}$, and when contracted with the lepton tensor will be proportional to the square of lepton mass, making their individual contributions negligible for electron and muon antineutrinos. However, they are present in "full-model." A detailed discussion over the individual contributions of all the diagrams will be given later in this section.

The $\Lambda \pi$ final state on neutron and proton target are shown in Fig. 4. Apart from the individual contributions,

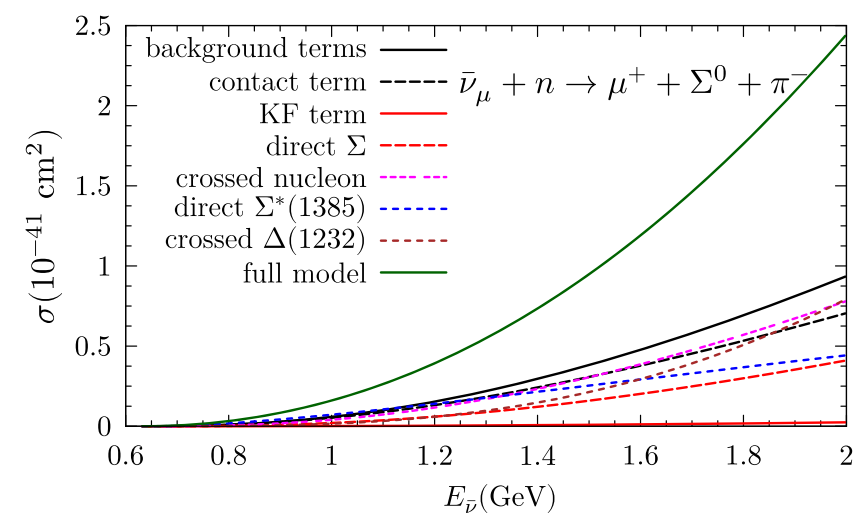

FIG. 5. Total cross sections for the $\Sigma^{0} \pi^{-}$and $\Sigma^{-} \pi^{0}$ production off neutrons. We present here results for $\Sigma^{0} \pi^{-}$production only. The results for the $\Sigma^{-} \pi^{0}$ are identical as the hadron amplitude is the same up to a relative sign (see Appendix). We also present individual contributions of some of the diagrams following Fig. 4. 

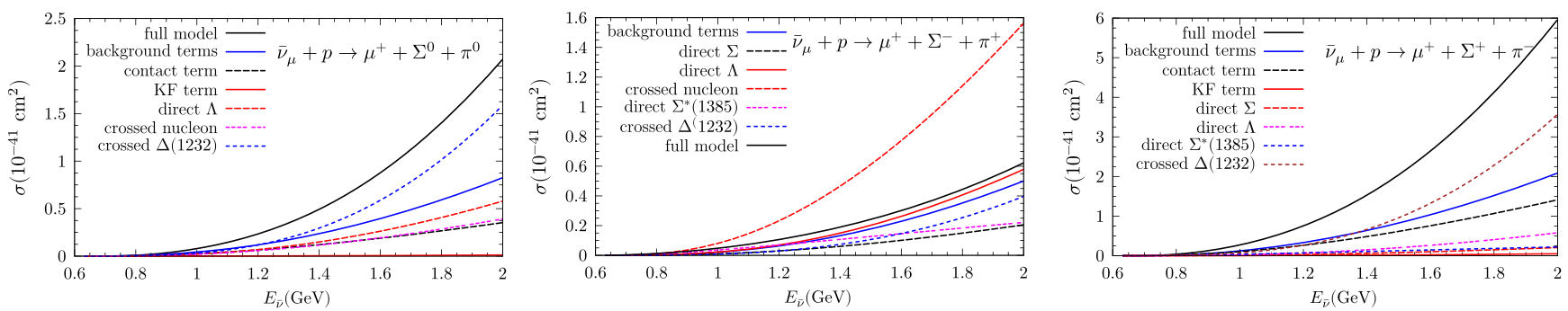

FIG. 6. Total cross sections for the $\Sigma$ hyperon production off protons, $\Sigma^{0} \pi^{0}$ on the left panel, $\Sigma^{-} \pi^{+}$on the middle one and $\Sigma^{+} \pi^{-}$on the right. The individual contribution is also shown, similar to Fig. 4.

we also present results for the background terms, where we add all the Feynman amplitudes of Fig. 1(a) coherently. We find that the background terms are comparable with the resonance contribution. One particular feature for the $\Lambda \pi$ production is that the cross section off the neutron target is exactly twice as that for proton target; see Appendix for the $\mathrm{SU}(3)$ relationships derived for the different amplitudes (hadronic currents).

A similar trend can be found for $\Sigma \pi$-production cross sections off neutrons, as depicted in Fig. 5. In this case, the possible $\Sigma \pi$-final states are $\Sigma^{0} \pi^{-}$and $\Sigma^{-} \pi^{0}$. However, it turns out that the cross section for both channels is exactly identical and is shown for one of the channels $\left(\bar{\nu}_{\mu}+n \rightarrow \mu^{+}+\Sigma^{0}+\pi^{-}\right)$. This can be understood from their isospin relations as given in Appendix, where the modulus of the isospin factors are the same for both the channels. While the individual diagrams contribute similarly to $\Lambda \pi$ production, the full model grows faster for the $\Sigma \pi$ reaction.

The relative size of the contributions of many mechanisms depicted in Figs. 1(a) and 1(b) can be understood in terms of their couplings alone, given by the constants $\mathcal{A}_{i}^{N \rightarrow Y \pi}$ of Tables III and IV. For instance, the smallness of the KF contributions in the reaction channels producing $\Sigma$ hyperons (Figs. 5 and 6) can be explained because their cross sections are proportional to the square of $(D-F)$, while for the reactions producing $\Lambda$ hyperons, these are proportional to the square of $(D+3 F)$, which is much larger. Further, if we compare $\Lambda \pi$ with $\Sigma \pi$ final states close to threshold energies, $\Lambda \pi$ cross section is higher than that of $\Sigma \pi$ as $M_{\Lambda}<M_{\Sigma}$, thus allowing a larger phase space for the same antineutrino energies. However, the overall contribution of KF diagram is relatively low, as the virtual $K$ in the KF diagram is carrying a four-momentum which is highly off-shell,

$$
p_{K}^{2}=\left(p-p_{Y}\right)^{2} \leqslant\left(M-M_{Y}\right)^{2} \ll M_{K}^{2},
$$

which also suppresses its contribution.

In fact, if one looks at the studies carried out in Refs. [72-74], this kind of contributions is more sizeable when the mass of the exchanged meson is lighter, as it is the case of the $\pi P$ diagrams with respect to the $\eta P$ ones, if one inspects some of the figures depicted in Refs. [72-74].

Next, we explore the crossed-nucleon diagrams. In general, the crossed-nucleon diagrams are important because of two main reasons: the constants of the diagrams $\mathcal{A}_{\mathrm{u}-\mathrm{N}^{\prime}}^{N \rightarrow Y \pi}$ are proportional to $(D+F)$ coupling coming from the $N N^{\prime} \pi$ vertex (see Table III), which is also large; and because the four-momentum squared carried by the intermediate nucleon is closer to its squared mass, given that the mass of the final $\pi$ meson is light (see Eq. (42) with $M_{\Delta}^{2}$ replaced by $M^{2}$ ). Therefore, in this case, the difference in the intermediate nucleon propagator, $\left(p-p_{m}\right)^{2}-M^{2}$, is smaller in absolute value than for the crossed- $\Delta$ propagator. The relative size of the crossed-diagrams for the different channels can be understood using Table III along with Tables I and II. For example, in Fig. 6, the ratio $\sigma_{u-N^{\prime}}\left(\Sigma^{0} \pi^{0}\right): \sigma_{u-N^{\prime}}\left(\Sigma^{-} \pi^{+}\right)$is $1: 4$, due to the square of constants $\mathcal{A}_{i}$ and the square of the ratio of vector and axialvector transition form factors for $p \rightarrow \Sigma^{0}$ and $n \rightarrow \Sigma^{-}$in Tables I and II. Something similar happens with the neutron induced $\Sigma \pi$ reactions of Fig. 5, but in this case the factors compensate, giving the same contribution (1:1 ratio) to the cross section.

In the $s$-channel we find that, normally, the direct $\Lambda$ contributions are larger than the direct $\Sigma$ ones off protons by a factor $\sim 3$ when both diagrams are present in the same reaction channel. This can be more or less understood because $\frac{D}{\sqrt{3}} \sim F$ and if one neglects (which is not a bad approximation for the vector form factors) the contribution of the charge $f_{1}^{n}\left(q^{2}\right)$ [certainly not good for the magnetic $f_{2}^{n}\left(q^{2}\right)$ ] form factor, the ratio of direct $\Lambda$ over direct $\Sigma$ is roughly $\left(\frac{D}{F}\right)^{2} \sim 3$. Of course, the pure axial-vector contribution and the interference vector-axial in those diagrams seem to have a trend to cancel because otherwise, the ratio $3: 1$ would not be so accurate as it happens to be.

The contribution of direct $\Sigma^{*}$ resonance channel is very important for the final $\Lambda \pi$ production reactions of Fig. 4, and gradually decreases for $\Sigma \pi$ production off neutrons (Fig. 5) and off protons (Fig. 6). The reason is two-fold: on the one hand, the ratio $\mathcal{A}_{\mathrm{s}-\Sigma^{*}}^{n \rightarrow \Sigma \pi}: \mathcal{A}_{\mathrm{s}-\Sigma^{*}}^{n \rightarrow \Lambda \pi^{-}}$is $1: \sqrt{3}$, and hence a factor 3 of reduction in the cross section is obtained off the neutron channels; similarly, for the reaction off protons 
a factor 6 of reduction in the cross section is found. Additionally, on the other hand, at low energies, the $\Lambda \pi$ production channel dominates over $\Sigma \pi$ because of the threshold effect. This threshold effect, together with smaller couplings for $\Sigma \pi$ channels, reduces the contribution of the $\Sigma^{*}$ resonance for the final production of $\Sigma \pi$.

On the other hand, the crossed $\Delta$ diagrams are important for the $\Sigma \pi$ reaction channels, especially when induced off protons (see Fig. 6). In fact, from Table IV the cross sections for the channels $p \rightarrow \Sigma^{+} \pi^{-}, p \rightarrow \Sigma^{0} \pi^{0}, n \rightarrow \Sigma \pi$ and $p \rightarrow \Sigma^{-} \pi^{+}$are found in the relative ratios $9: 4: 2: 1$, respectively.

In general, the interferences between the different mechanisms (diagrams) are significant and destructive, except for the $p \rightarrow \Sigma^{+} \pi^{-}$channel, see Fig. 6. For all other channels under consideration, we find that the interferences are important and reduce the total cross section compared with the incoherent sum of the singled-out contributions. In some cases, like in the reaction $\bar{\nu}_{\mu}+p \rightarrow \mu^{+}+\Sigma^{-}+\pi^{+}$ (see Fig. 6) a single mechanism is much larger than the total cross section. Similar results are found for the $\Lambda \pi$ production, as might be seen in Fig. 4. Here we must point out that the chiral Lagrangian fixes the relative sign between all (nonresonant) diagrams, at least close to the threshold.

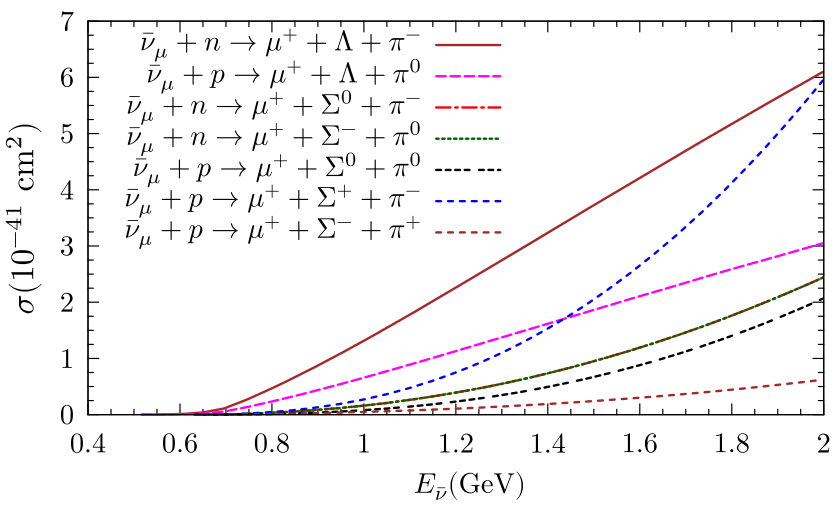

FIG. 7. Plot of the total cross sections for $Y \pi$ production off nucleons induced by muon antineutrinos as a function of the antineutrino energy in the LAB frame.
In Fig. 7, we present the total cross sections for the full model corresponding to all the possible $Y \pi$ channels induced by muon antineutrinos off nucleons as a function of the antineutrino energy in the LAB frame. It is interesting to see that the total cross sections have the same order of magnitude as those of the single $K$ and $\bar{K}$ production $(1 K / \bar{K})$ cross sections off nucleons studied in Refs. [72,73]. While the $1 K / \bar{K}$ cross sections are smaller than the single pion cross sections because of the smallness of the Cabibbo angle; the $Y \pi$ cross section misses the strong $\Delta$ (1232)-like mechanism, apart from the threshold effect.

Finally, in Fig. 8 we show the comparison between the electron antineutrino and muon antineutrino induced $Y \pi$ production total cross sections as a function of the antineutrino energy in the LAB frame. As expected, the cross sections for electron antineutrinos are larger than their muon counterparts because of their lower production thresholds due to the smallness of the final electron mass than the muon one. A similar trend is found for all other reaction channels.

\section{B. Comparisons with other models}

This work presents a detailed analysis of the $Y \pi$ production cross section induced by antineutrinos. To the best of our knowledge, our calculations are one of the first in studying these processes. However, there are independent calculations where the authors calculate the quasifree production of an on-shell $\Sigma^{* 0}(1385)$ resonance [63]. In order to make a comparison with the $\Sigma^{* 0}(1385)$ production, we consider only the s-channel $\Sigma^{*}$ diagram. To compare the production cross sections of specific $Y \pi$ channels, we have taken into account the primary decay channels of $\Sigma^{*}: \Lambda \pi^{0}$ and $\Sigma \pi$ with branching ratios $87 \%$ and $11.7 \%$ respectively [91]. Further, the inclusive $\Sigma \pi$ decay channel may have different candidates, viz, $\Sigma^{ \pm} \pi^{\mp}$ and $\Sigma^{0} \pi^{0}$. The individual contribution of these final states can be obtained by multiplying by the appropriate (square of) Clebsch-Gordan coefficients, which is zero for $\Sigma^{0} \pi^{0}$ and $\frac{1}{2}$ for $\Sigma^{ \pm} \pi^{\mp}$. The results are shown in Fig. 9. In the left panel of Fig. 9, where the two models show a remarkable coincidence, the solid lines correspond to our model, while
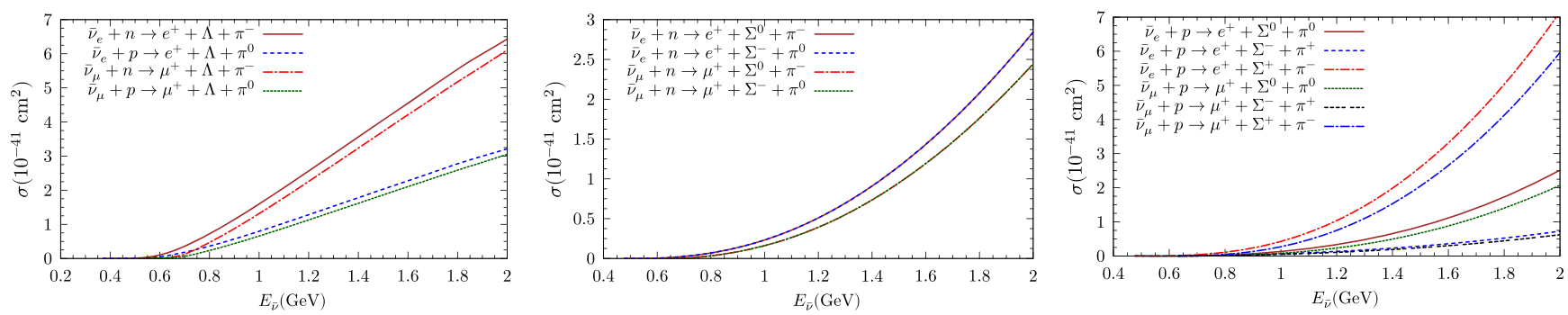

FIG. 8. Comparison between electron antineutrino and muon antineutrino induced total cross sections off nucleons in terms of the antineutrino energies in the LAB frame. In the left panel we display the $\Lambda \pi$ reaction channels. In the middle panel we show the $\Sigma \pi$ production channels off neutrons. Finally, in the right panel we plot the $\Sigma \pi$ reactions off protons. 

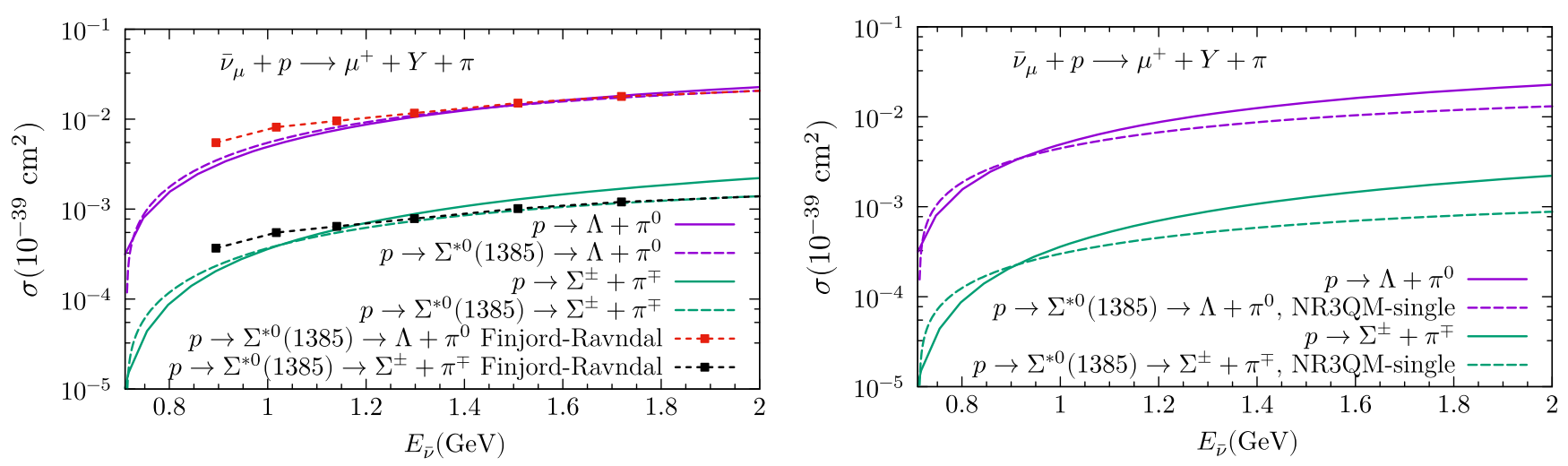

FIG. 9. Comparison for the reaction of Cabibbo suppressed single pion production off protons with the mechanism of intermediate $\Sigma^{* 0}$ alone. We compare with the results obtained in Ref. [63], where the authors calculate the quasi-free production of an on-shell $\Sigma^{* 0}$ off protons induced by muon antineutrinos. Solid lines represent our model with only s- $\Sigma^{*}$ reaction mechanism, while dashed lines are the results of Ref. [63] for the V-A approach(left panel); and the NR3QM-single approach(right-panel). On the left panel, we also display as dotted lines with filled squares the results of Ref. [64].
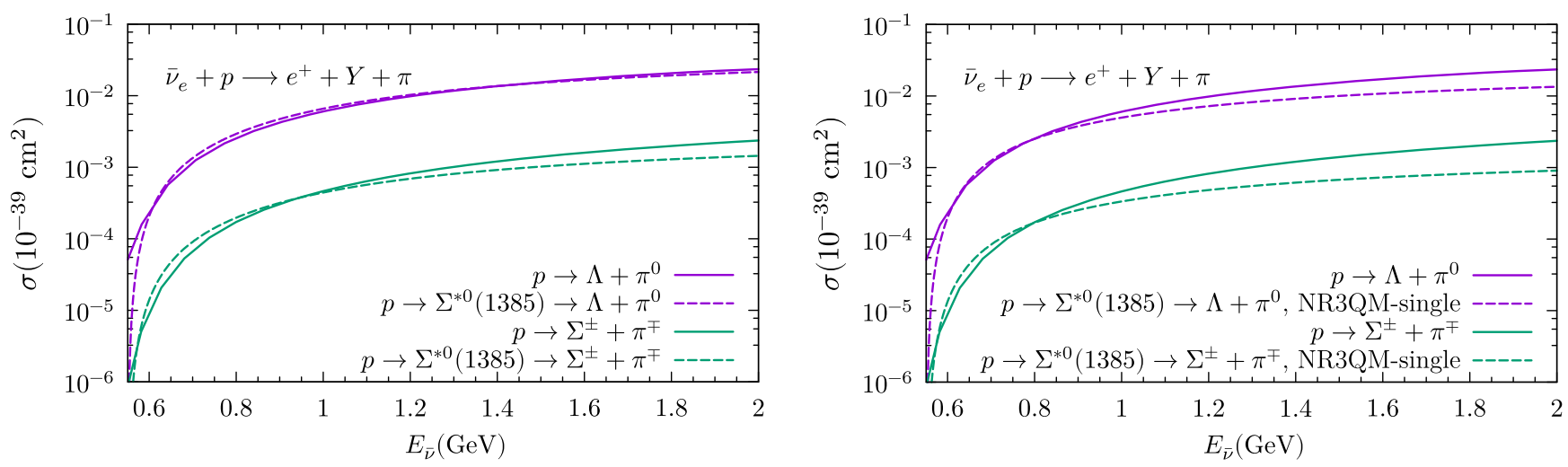

FIG. 10. Same as Fig. 9 but for the reactions induced by electron antineutrinos off protons. Panels and lines have the same meaning as in Fig. 9.

the dashed lines are those of Ref. [63] with the V-A approach. They use an axial mass $M_{A}=1.05 \mathrm{GeV}$ for the axial form factor $C_{5}^{A}\left(q^{2}\right)$, as being used in the present model. ${ }^{5}$ Off-shell effects present in our model show a slight discrepancy at the highest energies shown in the left panel for the $\Sigma^{ \pm} \pi^{\mp}$ production channel. Also on this panel we show as dotted lines with filled squares the corresponding results of Ref. [64], where again the coincidence for the decay channel $p \rightarrow \Sigma^{* 0}(1385) \rightarrow \Lambda \pi^{0}$ at the higher energies shown in the plot is remarkable.

In the right panel of Fig. 9, we compare our results with the nonrelativistic 3 quark model (NR3QM-single) discussed in Ref. [63]. In this case, the discrepancies are larger at smaller antineutrino energies; however, this is expected as the cross sections calculated within the NR3QM-single

\footnotetext{
${ }^{5}$ The readers should note that this axial mass used in the nucleon-to-resonance transition axial-vector form factor $C_{5}^{A}$ is different from the axial mass appearing in Eq. (23) for the nucleon axial form factor.
}

approach were already smaller than those calculated within the V-A approach (see Fig. 10 in Ref. [63]). Nonetheless, we find that the cross sections are of the same order of magnitude, even when comparing with the most unfavorable approach.

Figure 10 shows a similar comparison as in Fig. 9 but with the results of Ref. [63] for the reactions induced by electron antineutrinos off protons. In this latter case, the thresholds are a bit lower, but the general features found in Fig. 9 remain the same. One should note that the comparison on the left panel of Fig. 10 with the V-A approach of Ref. [63] is expected as both models are identical, except for the off-shell treatment of $\Sigma^{* 0}(1385)$ resonance. However, on the right panel of Fig. 10, the agreement with the NR3QM-single approach is more inadequate as it already was in the right panel of Fig. 9.

Finally, in Fig. 11 we show the comparison between the results of the total cross sections for the three charge $\Sigma \pi$ states production channels off protons in our model (solid lines) versus the results of Ref. [62] (short-dashed lines). 


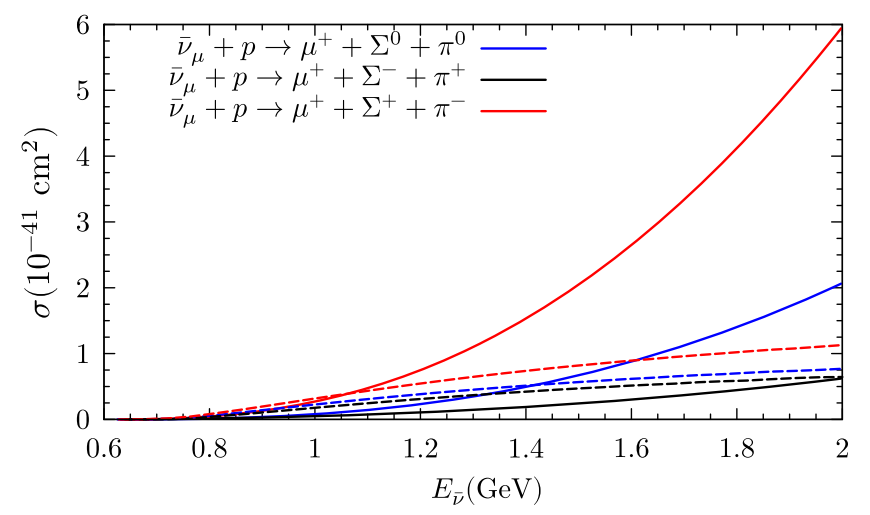

FIG. 11. Comparison between the total cross sections for the three $\Sigma \pi$ reaction channels for our model (solid lines) and that of Ref. [62] (short-dashed lines).

The model of Ref. [62] is based on a chiral unitary approach where all the meson-baryon pairs with $S=-1$ produced in a primary $\mathrm{CT}, \mathrm{KP}$ or meson-in-flight (MF) diagram are allowed to interact in a coupled channels approach to dynamically generate the $\Lambda(1405)$ resonance by solving the Bethe-Salpeter equation with an interaction potential derived from the lowest-order chiral Lagrangian of Eq. (15).

If we inspect Fig. 11, we observe that the total cross sections derived in our model are generally much larger than those of Ref. [62], especially important is the enhancement for the $\Sigma^{+} \pi^{-}$channel, which amounts to almost a factor 6 at $E_{\bar{\nu}}=2 \mathrm{GeV}$. More moderate is the enhancement for the $\Sigma^{0} \pi^{0}$ channel, and for the $\Sigma^{-} \pi^{+}$one, where our cross section is smaller than its counterpart of Ref. [62]. Nonetheless, close to threshold the three cross sections are larger in Ref. [62] than in our model, even although we explicitly incorporate a resonant diagram with a $\Sigma^{*}(1385)$ resonance which is below the $\Lambda(1405)$ resonance and above the $\Sigma \pi$ threshold. This clearly means that the $\Lambda(1405)$ plays an important role in the description of these reactions close to threshold for the $\Sigma \pi$ production channels. Probably the reason for this is that the $\Lambda(1405)$ appears in s-wave coupled channels and these are going to be much more important close to threshold. However, the $\Sigma^{*}(1385)$ is a p-wave resonance like the $\Delta$, and its contribution, already small due to its couplings (as shown in Fig. 6) for these reactions, starts to contribute more at higher antineutrino energies.

However, the $\Lambda(1405)$ resonance is not going to play any role for the $\Lambda \pi^{0}$ production off protons because it appears in the $I=0$ channel and the final one has $I=1$. In the coupled channel approach of Ref. [62] there is the possibility of producing a final $\Lambda \pi^{0}$ through a loop of $\bar{K} N$ intermediate states produced in the weak vertices coupled to $I=1$. And indeed, these $\bar{K} N$ states couple directly (at the level of $V_{i j}$ in the nomenclature of Ref. [92]) to $\Lambda \pi^{0}$ (see $C_{i j}$ coefficients of Table I of [92] for the couplings of the two $\bar{K} N$ states to $\Lambda \pi^{0}$ ). Based on these arguments, we think the most reliable and unaffected by the presence of higher lying strange resonances are those reaction channels with a $\Lambda$-particle as a final state.

One similarity between the results of Ref. [62] and ours is that the order of the channels with larger cross sections matches significantly, i.e., the cross section for $\Sigma^{+} \pi^{-}$ production is larger than that for $\Sigma^{0} \pi^{0}$ followed by $\Sigma^{-} \pi^{+}$ production, and the above trend is consistent in both approaches. This extends the reliability in the present model.

Also note that in the calculations of Ref. [62], a nonrelativistic reduction of the amplitudes was carried out. These approximations can also have an impact in the differences observed in the size of the cross sections for the same range of antineutrino energies shown in Fig. 11. However, we cannot at the present moment quantify how much of the difference comes from the nonrelativistic approximation and/or from other relevant ingredients present in the model of Ref. [62] and absent in ours, or vice versa.

Finally, it is also worth noticing that the way these cross sections rise in our model is very similar to how the crossed or u-channel diagrams do it, especially the crossed $\Delta$ diagrams plotted in Fig. 6, which are very relevant by themselves, especially for the $\Sigma^{+} \pi^{-}$and $\Sigma^{0} \pi^{0}$ reaction channels, which are those with the largest cross sections. This could point to the importance of crossed diagrams, not only for $\Delta$ intermediate states, but also for $N^{*}$ resonances not considered here.

\section{Flux-integrated total cross sections}

In this work, we have also estimated the flux-folded total cross sections for antineutrino fluxes of several experiments like MiniBooNE [93], SciBooNE [94], T2K [95,96], and Minerva [97]. The energy dependence of these fluxes is shown in Fig. 12. We choose antineutrino fluxes that peak at intermediate energies, i.e., $\left\langle E_{\bar{\nu}}\right\rangle \simeq 1-3 \mathrm{GeV}$. At these energies, the four-momentum transfers are expected to be low enough to carry chiral expansions, making the present model more reliable.

The definition of the flux-integrated total cross section, $\langle\sigma\rangle$, for a given antineutrino flux $\Phi\left(E_{\bar{\nu}}\right)$ of some experiment, can be obtained as

$$
\langle\sigma\rangle=\frac{\int_{E_{\bar{\nu}}^{\mathrm{th}}}^{E^{\max }} \Phi\left(E_{\bar{\nu}}\right) \sigma\left(E_{\bar{\nu}}\right) d E_{\bar{\nu}}}{\int_{0}^{E^{\max }} \Phi\left(E_{\bar{\nu}}\right) d E_{\bar{\nu}}} .
$$

In Eq. (44), the lower limit in the integral of the numerator can be also zero, but it is not necessary, because the total cross section $\sigma\left(E_{\bar{\nu}}\right)$ is zero for $E_{\bar{\nu}}<E_{\bar{\nu}}^{\text {th }}$, where $E_{\bar{\nu}}^{\text {th }}$ is the threshold antineutrino energy in the LAB frame for the reaction to take place. Its expression is given by 

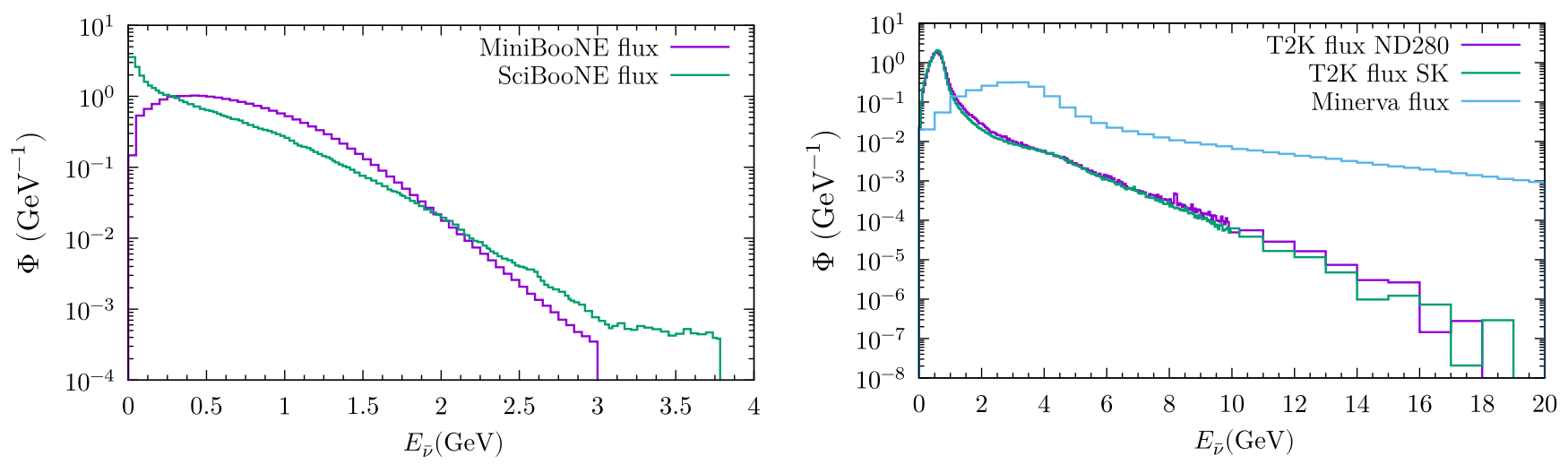

FIG. 12. Fluxes from different experiments. On the left panel, the $\bar{\nu}_{\mu}$ fluxes from MiniBooNE [93] and SciBooNE [94]. On the right panel, the T2K fluxes at the near detector ND280 and at the Super-Kamiokande far detector [95,96], and the enriched $\bar{\nu}_{\mu}$ Minerva flux [97]. The fluxes are normalized to their total flux, i.e., the integral of the fluxes shown in this figure is 1 .

$$
E_{\bar{\nu}}^{\mathrm{th}}=\frac{\left(M_{Y}+m_{\pi}+m_{l}\right)^{2}-M^{2}}{2 M}
$$

thus giving $E_{\bar{\nu}}^{\text {th }} \simeq 0.515 \mathrm{GeV}$ for final $\Lambda$ production and $E_{\bar{\nu}}^{\text {th }} \simeq 0.630 \mathrm{GeV}$ for final $\Sigma$ production induced by muon antineutrinos. While, the $E^{\max }$ depend upon the flux and their values are $20 \mathrm{GeV}$ for Minerva and T2K, 3 and $4 \mathrm{GeV}$ for MiniBooNE and SciBooNE, respectively.

In Table $\mathrm{V}$ we show the flux-folded total cross sections for muon antineutrinos fluxes from different experiments: MiniBooNE [93], SciBooNE [94], T2K [95,96], and Minerva [97].

The T2K (both at the near detector ND280 and at SuperKamiokande one) and Minerva fluxes have larger tails ranging up to $20 \mathrm{GeV}$. Our model, which is based on a chiral expansion, is not going to be reliable for these higher energies, where high momentum transfers and high invariant masses become accessible with the increase of the antineutrino energies. In order to overcome this difficulty, we have put a constraint on the final invariant hadronic mass, $W<1.4 \mathrm{GeV}$. This solves two problems: on the one hand, we are sure that higher lying strange resonances above the $\Sigma^{*}(1385)$, such as the $\Lambda(1405)$ (which has been shown in Fig. 11 to contribute significantly to the $\Sigma \pi$ production channel near threshold), are not going to contribute for these kinematically constrained total cross sections; on the other hand, the total cross sections when the cut in the invariant mass is imposed, do not grow rapidly and hence allow to calculate a well-defined fluxaveraged total cross section with the low energy fluxes like T2K and Minerva (low energy mode). In addition, this cut has also a virtue, because it can be also experimentally imposed, thus rejecting the $Y \pi$ events with measured invariant masses $W>1.4 \mathrm{GeV}$.

In order to analyze the results shown in Table V, it is important to remark that the flux-folded total cross sections do not depend on the total flux, because they are normalized to it. They depend basically on the shape of the flux and where they are mostly peaked and if their tails are longer or shorter. And also on how large is the total cross section in the zone where the flux is sizeable. With this in mind, we can understand the calculations shown in Table V.

The first comparison we analyze is between the fluxaveraged cross sections for MiniBooNE [93] and SciBooNE [94] experiments. Note that the flux taken for MiniBooNE, Ref. [93], corresponds to the antineutrino enhanced sample, while the flux taken from Fig. 1 of Ref. [94] corresponds also to the $\bar{\nu}_{\mu}$ flux, but in this case

TABLE V. Flux-folded total cross sections for $\bar{\nu}_{\mu}$ fluxes from different experiments, in units of $10^{-42} \mathrm{~cm}^{2}$. The cut in the final invariant hadronic mass $W \leqslant 1.4 \mathrm{GeV}$ has been applied to the calculations for the T2K and Minerva fluxes. The uncertainties are in the last significant figure.

\begin{tabular}{lccccc}
\hline \hline Reaction & MiniBooNE & SciBooNE & T2K ND280 & T2K SK & Minerva \\
\hline $\bar{\nu}_{\mu}+p \rightarrow \mu^{+}+\pi^{0}+\Lambda$ & 3.42 & 1.95 & 2.17 & 1.68 & 23.8 \\
$\bar{\nu}_{\mu}+n \rightarrow \mu^{+}+\pi^{-}+\Lambda$ & 6.84 & 3.90 & 4.33 & 3.36 & 47.7 \\
$\bar{\nu}_{\mu}+p \rightarrow \mu^{+}+\pi^{0}+\Sigma^{0}$ & 0.935 & 0.713 & 0.0684 & 0.0546 & 0.623 \\
$\bar{\nu}_{\mu}+p \rightarrow \mu^{+}+\pi^{-}+\Sigma^{+}$ & 2.88 & 2.13 & 0.290 & 0.231 & 2.85 \\
$\bar{\nu}_{\mu}+p \rightarrow \mu^{+}+\pi^{+}+\Sigma^{-}$ & 0.369 & 0.254 & 0.111 & 0.0887 & 1.36 \\
$\bar{\nu}_{\mu}+n \rightarrow \mu^{+}+\pi^{-}+\Sigma^{0}$ & 1.38 & 0.954 & 0.263 & 0.211 & 2.96 \\
$\bar{\nu}_{\mu}+n \rightarrow \mu^{+}+\pi^{0}+\Sigma^{-}$ & 1.38 & 0.954 & 0.263 & 0.211 & 2.96 \\
\hline \hline
\end{tabular}


this is not the larger component of the flux, because the latter is the muon neutrino component.

From Table V, we find that the results from MiniBooNE and SciBooNE differ significantly, though the fluxes do not look strikingly different in nature, see left panel of Fig. 12. The reason for the differences is that the SciBooNE flux peaks at antineutrino energies below the threshold for the reaction to take place. However, the SciBooNE flux has a longer tail which decreases a bit slowly than the MiniBooNE one. The flux averaged cross sections are always higher for MiniBooNE than for SciBooNE because the MiniBooNE flux is larger in the region between 0.5 and $2 \mathrm{GeV}$, and the presence of the SciBooNE tail has little importance (specially for the $\Lambda \pi$ production channels) even although in this region the cross section is growing (without the cut in the hadronic invariant mass).

It is worth noting that there is a difference between the averaged cross sections for the reactions $p \rightarrow \Lambda \pi^{0}$ and $p \rightarrow$ $\Sigma^{+} \pi^{-}$in both experiments. The first reaction has a higher flux-folded cross section with the MiniBooNE flux, while the opposite happens with the SciBooNE one. The reason for this has to be looked for in the behavior of the cross sections for these two reactions in the higher energy tails of the fluxes. Indeed, the $p \rightarrow \Sigma^{+} \pi^{-}$cross section grows clearly steeper with the antineutrino energy than the $p \rightarrow$ $\Lambda \pi^{0}$ one does, as can be seen in Fig. 7. Therefore, the SciBooNE slowly decreasing tail has a compensating effect for the $p \rightarrow \Sigma^{+} \pi^{-}$reaction, because in the region of this tail, the cross section for $p \rightarrow \Sigma^{+} \pi^{-}$is much larger than that for the $p \rightarrow \Lambda \pi^{0}$ channel, thus making the flux-folded $p \rightarrow \Sigma^{+} \pi^{-}$cross section the second in magnitude for the SciBooNE flux, while it was the third in size with the MiniBooNE one.

For the flux-folded total cross sections with the T2K near detector ND280, and Super-Kamiokande far detector fluxes [95,96], and with the Minerva flux [97], we have applied the cut $W \leq 1.4 \mathrm{GeV}$ in the final hadronic invariant mass.
This cut has the obvious effect of reducing the size of the total cross sections, as can be observed in Fig. 13. However, the reduction in size is much more prominent for the $\Sigma \pi$ reactions than for the $\Lambda \pi$ ones. The reason for this behavior is because the cut in the invariant mass is much closer to the threshold for $\Sigma \pi$ production $\left(W_{\mathrm{th}}^{\Sigma \pi}=M_{\Sigma}+m_{\pi} \simeq\right.$ $1.33 \mathrm{GeV}$ ) than it is for the $\Lambda \pi$ production channels $\left(W_{\mathrm{th}}^{\Lambda \pi}=M_{\Lambda}+m_{\pi} \simeq 1.25 \mathrm{GeV}\right)$. In fact, obviously, if the applied cut had been below the $\Sigma \pi$ threshold, all these cross sections would have been exactly zero.

Therefore, this huge reduction in the size of the total cross sections for the $\Sigma \pi$ reaction channels when the cut in the invariant mass is applied explains why the fluxaveraged total cross sections with the T2K and Minerva fluxes are so small if compared with their $\Lambda \pi$ counterparts in Table V. The reduction due to the cut in the invariant mass amounts to a roughly one order of magnitude smaller for the $\Sigma \pi$ reactions. There is even a reaction channel, $p \rightarrow \Sigma^{0} \pi^{0}$, where the reduction of the cross section due to the cut in the invariant mass is specially significant, as it can be observed in the right panel of Fig. 13, because it is the smallest cross section of the $\Sigma \pi$ channels, while this was not the case when there was no cut in the final hadronic invariant masses. In fact, for this particular reaction channel, the reduction in the flux-averaged total cross sections is already two orders of magnitude than for the $\Lambda \pi$ reactions. For this reason, we have plotted in logarithmic scale the cross sections for the $\Sigma \pi$ channels when comparing them with the cut and without it in the right panel of Fig. 13, because in a linear vertical scale the cross sections with the cut in the invariant mass were almost not visible.

Of particular curiosity is the similarity of the flux-folded total cross sections for the $p \rightarrow \Sigma^{+} \pi^{-}$channel and the $n \rightarrow$ $\Sigma \pi$ (both final charge channels have exactly the same cross section) one when the cut in the invariant mass is applied, even for so different fluxes such as those of T2K and
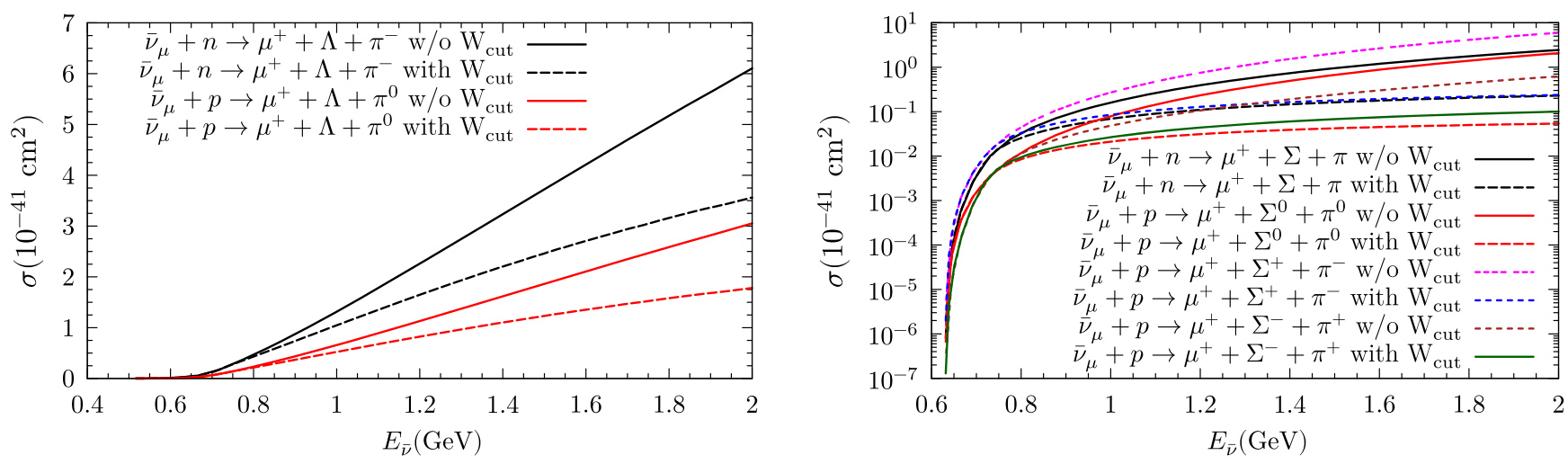

FIG. 13. Plots of the total cross sections for the $Y \pi$ production as a function of the antineutrino energy with the effect of the kinematic cut in the final hadronic invariant mass $W \leq 1.4 \mathrm{GeV}$. In the left panel we show the results for $\Lambda \pi$ production, while in the right one we display those for the $\Sigma \pi$ case. For this latter case the y-axis is logarithmic because of the huge reduction in the cross sections when the cut $W \leq 1.4 \mathrm{GeV}$ is imposed. 
Minerva, which are peaked at totally different antineutrino energies and have really different tails, as shown in the right panel of Fig. 12. However, as the reduced total cross sections (due to the cut) for both channels are so similar (compare blue and black dashed lines in the right panel of Fig. 13), their flux-averaged total cross sections shown in Table $\mathrm{V}$ for the $\mathrm{T} 2 \mathrm{~K}$ and Minerva fluxes are also very similar. Nonetheless, the flux-averaged cross section for the $p \rightarrow \Sigma^{+} \pi^{-}$channel is larger than those of the $n \rightarrow \Sigma \pi$ ones for the $\mathrm{T} 2 \mathrm{~K}$ fluxes because these are peaked below $1 \mathrm{GeV}$, where the cross section for the $p \rightarrow \Sigma^{+} \pi^{-}$production channel is a bit larger. For the Minerva flux the result is the opposite because this flux is peaked around $3 \mathrm{GeV}$, although the differences, as discussed, are really minor.

It is also worth mentioning that even although both T2K fluxes at near and far detectors are almost equal (see the right panel of Fig. 12), the flux-folded total cross sections are systematically smaller when convoluted with the flux at the SK detector for all the reactions (the reader can compare the numbers in the fourth and fifth columns of Table V). The reason for this has to be searched in the slightly smaller tail of the T2K flux at SK, compared with that at the ND280, especially in the region between 1 and $4 \mathrm{GeV}$ of muon antineutrino energies, where its contribution is still relevant for the flux-integrated total cross section.

Finally, the large numbers for the flux-averaged total cross sections with the Minerva flux shown in the last column of Table $\mathrm{V}$, especially for the $\Lambda \pi$ production channels, and if compared with the same numbers for the T2K fluxes, can be explained because the Minerva flux is peaked around $3 \mathrm{GeV}$, where the cross sections are much larger than in the region where the T2K fluxes are peaked. And, additionally, the larger and slowly decreasing tail of the Minerva flux (solid cyan line on the right panel of Fig. 12) has also a very important role in the enhancement of the flux-convoluted total cross sections for this experiment, in comparison with the results obtained for $\mathrm{T} 2 \mathrm{~K}$.

\section{CONCLUSIONS}

In this work we have studied the Cabibbo suppressed single pion production off nucleons induced by antineutrinos. This process, which is the strangeness-changing counterpart of the largely studied single pion production without change of strangeness, has been very scarcely analyzed so far. In these reactions, the final pion is emitted along with a $\Sigma$ or $\Lambda$ hyperon.

It is well-known that its Cabibbo enhanced counterpart is largely driven by the weak excitation of the $\Delta$ resonance, therefore we have also considered in our model the relevant $(S=-1) \Sigma^{*}(1385)$ resonance, belonging to the same decuplet as the $\Delta$. In fact, we have found that this mechanism is indeed the dominant one for the $\Lambda \pi$ reactions, but of minor importance for the $\Sigma \pi$ channels. We have also found that crossed $\Delta$ or nucleon-pole diagrams are also important, especially for some of the $\Sigma \pi$ reactions.
This could indicate that the inclusion of $N^{*}$ resonances in the u-channel can be necessary, but the absence of experimental data on these reactions refrains us from doing any categorical statement about this.

We have also compared our results with others found in the recent and past literature. The main conclusion is that the $\Lambda(1405)$ resonance plays an important role close to threshold, especially due to its S-wave character, in comparison with the P-wave character of the $\Sigma^{*}$ resonance. However, when one goes to higher antineutrino energies, other mechanisms and higher partial waves start to play an important role. Because the $\Lambda(1405)$ is an isospin 0 state, we can say that this resonance is not going to have any impact in the $\Lambda \pi$ reactions (which are those with the largest total cross sections in our model up to antineutrino energies of $2 \mathrm{GeV}$ in the LAB frame) because there cannot be any coupling due to conservation of strong isospin. Therefore, our most reliable results are expected to be those producing final $\Lambda \pi$ hadrons for the range of antineutrino energies explored in this work.

We have also studied the flux-convoluted total cross sections of these reaction channels with the antineutrino fluxes of past (MiniBooNE, SciBooNE) and current (T2K near and far detectors, Minerva) neutrino oscillation and scattering experiments. The numbers obtained for these flux-folded total cross sections, and given in Table V, together with the conclusions drawn for the same observable with the antineutrino Minerva flux (also with invariant mass cut) in Table III of Ref. [75], indicate that these cross sections can be measured in Minerva experiment, especially the cross sections for final $\Lambda \pi$ production.

Compared to $\Delta S=0$ pion production, the smallness of cross section makes $\pi Y$ processes hard to detect. This means that the feasibility of detecting these channels in experiments is also limited. However, in some recent experiments like Minerva [45,58,59], the reconstructions of the incident neutrino/antineutrino energy and the invariant hadronic mass were shown to be feasible for semiinclusive samples containing charged pions $[45,58]$ and a single neutral pion [59] in charged current muon neutrino and antineutrino scattering off hydrocarbon $(\mathrm{CH})$ target, respectively. In fact, the experimental data for the total cross section as a function of the antineutrino energy for the single neutral pion sample was shown in Fig. 10(b) of Ref. [45]. In the lowest energy bin, the cross section has a value of $19.8 \times 10^{-41} \mathrm{~cm}^{2} /$ nucleon, although with more than $100 \%$ of uncertainty. For larger energy bins, the uncertainties are much smaller. Nevertheless, we can also provide our results for larger antineutrino energies, where the experimental data are expected to be less uncertain. The caveat here is that we have to apply the cut in the invariant mass to ensure that our model for the primary interaction is more reliable. Moreover, the cross section is comparable at these energies for the $\pi^{0}$ production channels; for example, see Fig. 7. Our values shown in this figure are about one 
order of magnitude smaller than that for the semi-inclusive process studied in [45]. Thus, with higher statistics we think they can be measured experimentally. Finally, FSI experienced by pions in nuclear targets can indeed distort the final signal, changing the identity of the final pion through mechanisms like charge exchange; however, they may get compensated by the secondary pions produced from hyperons. Detailed analysis must be required where our results may be used as the input for the effects like FSI.

The primary pions produced in the reactions studied here have a significant probability of being absorbed in the nucleus, but the hyperons are long-lived particles $\left(\tau \sim 10^{-10} \mathrm{~s}\right.$, except for the $\left.\Sigma^{0}\right)$ with small widths even in the nuclear medium $[67,70]$ (this is particularly true for the $\Lambda$ ) and exit the nucleus decaying weakly into secondary pions and nucleons. If these nucleons are below the experimental detection threshold (and therefore there is no way of reconstructing the invariant mass of the decaying hyperon), the final signal for the whole process could be indistinguishable from other mechanisms for pion production. This would contribute to the distortion of the tagging of the different processes leading to pion production in antineutrino-nucleus scattering.

It is also worth mentioning that, recently, a revival of detectors (basically high-pressure time projection chambers with adequate admixtures of argon and hydrogen-enriched gases such as methane [98]) with high-quality momentum resolution and using the technique of the transverse momentum imbalance [99] has emerged with the claim of being exquisite for the measurements of neutrino/ antineutrino-hydrogen cross sections, and the discrimination of these reaction channels from other background nuclides present in the target material. The good point of these detection techniques is to eliminate nuclear effects at the price of being able to detect only final charged particles. If finally, this kind of detector prevails, then it will be possible to study neutrino/antineutrino cross sections off free protons with high accuracies, such as some of the proposed and studied in this work, particularly those where all the final particles are charged.

Finally, we think that our model can be suitable to be implemented in the Monte Carlo event generators as the primary interaction, which can then be used as an input to simulate the propagation of the $\pi Y$ pair inside the nuclear medium after incorporating the relevant nuclear effects. Nonetheless, SU(3) breaking effects can also be applied to see its plausible outcomes on cross sections within some model based approaches like Ref. [100], already applied in the $K \Xi$ production channel studied in Ref. [75].

\section{ACKNOWLEDGMENTS}

The authors wish to thank Prof. Bing-Song Zou and Dr. Jia-Jun Wu for providing their theoretical results of their study for comparison with ours. We also want to specially thank Dr. Luis Alvarez-Ruso for providing the results of his work (together with his collaborators in that work) for comparison with our results in this paper. And we also wish to thank him for very fruitful discussions that have helped us to understand their results and their underlying model, in order to try to discuss as accurately as possible the implications of their results in comparison with ours. The authors also thank Dr. Justo Martín-Albo for drawing our attention to the new proposals for detection of pure (anti)neutrino-hydrogen events with high pressure time projection chambers. To carry out some of the numerical calculations of this work the authors have used the resources of the scientific computing cloud PROTEUS of the Instituto Interuniversitario Carlos I de Física Teórica y Computacional of the University of Granada, and therefore acknowledge this computational facility. This work has been partially supported by Spanish Ministry of Science under Grants No. FIS2017-85053-C2-1-P and No. PID2020-114767GB-I00, and by the Junta de Andalucia (Grant No. FQM-225). M. B. G. also acknowledges support from Spanish Ministry of Science under Grant No. PRE2018-083794. M. R. A thanks UGCBSR Startup Research Grant (F-NO. 30/2015/BSR), Government of India for partial support.

\section{APPENDIX: SU(3) RELATIONS BETWEEN THE AMPLITUDES}

In this Appendix we derive the relations between the amplitudes (currents) for the seven reaction channels discussed in this work using $\mathrm{SU}(3)$ group theoretical arguments.

First of all, we have to make the assignments between the physical states and the mathematical [or SU(3)] ones for the meson and baryon states of the octet before applying the Wigner-Eckart theorem. Besides, we have to identify the irreducible tensor operator belonging to the $\{8\}$ representation of SU(3) group that drives the strangeness-changing weak transition.

The strangeness-changing weak charged current (without the $V_{u s}$ Cabibbo-Kobayashi-Maskawa matrix element) carries "magnetic" quantum numbers of $\mathrm{SU}(3)\left(I, I_{3}, Y\right)=$ $\left(\frac{1}{2},-\frac{1}{2},-1\right)$, i.e., those quantum numbers of the $K^{-}$or the $\Xi^{-}$. This current operator can be written at the quark level as

$$
\begin{aligned}
j_{\Delta S=-1}^{\mu} & =\bar{Q} \gamma^{\mu}\left(1-\gamma_{5}\right)\left(F_{4}-i F_{5}\right) Q \\
& =-\sqrt{2} \bar{Q} K_{\left(\frac{1}{2},-\frac{1}{2},-1\right)}^{\mu\{8\}},
\end{aligned}
$$

where $K_{\left(\frac{1}{2},-\frac{1}{2},-1\right)}^{\mu\{8\}}=-\frac{1}{\sqrt{2}} \gamma^{\mu}\left(1-\gamma_{5}\right)\left(F_{4}-i F_{5}\right)$, with $F_{i}=\frac{\lambda_{i}}{2}$ (being $\lambda_{i}$ the Gell-Mann matrices). But in general $K_{\left(\frac{1}{2},-\frac{1}{2},-1\right)}^{\mu\{8\}}$ is an irreducible tensor current operator belonging to the $\{8\}$ representation of the $\mathrm{SU}(3)$ group carrying the $\mathrm{SU}(3)$ quantum numbers of this representation explicitly written in the subindex. Therefore, to this operator we can apply 
the Wigner-Eckart theorem of SU(3) [101]. Therefore, from here onwards we will work with this operator by assuming that we do not have quarks any longer and that the vector and axial-vector Dirac and Lorentz structure can be more complex than simply $\gamma^{\mu}\left(1-\gamma_{5}\right)$, which is the structure at the quark level only.

For simplicity in the notation, we will write the strangeness-changing current operator simply as

$$
j_{\mathrm{sc}}^{\mu} \equiv j_{\Delta S=-1}^{\mu}=-\sqrt{2} K_{\left(\frac{1}{2},-\frac{1}{2},-1\right)}^{\mu\{8\}},
$$

and we will calculate all the transition matrix elements driven by the above current between initial nucleon states and final $\Sigma \pi$ and $\Lambda \pi$ states. To this end, we have to fix the phases between the physical states and the mathematical ones for which the SU(3) Clebsch-Gordan coefficients have been calculated $[85,101]$ in order to appropriately use the Wigner-Eckart theorem. For the physical states we have in our study, this phase fixing convention for mesons and baryons is

$$
\begin{aligned}
|p\rangle & =\left|\{8\} ; \frac{1}{2}, \frac{1}{2}, 1\right\rangle & & |n\rangle=\left|\{8\} ; \frac{1}{2},-\frac{1}{2}, 1\right\rangle \\
\left|\Sigma^{+}\right\rangle & =-|\{8\} ; 1,1,0\rangle & & \left|\Sigma^{0}\right\rangle=|\{8\} ; 1,0,0\rangle \\
\left|\Sigma^{-}\right\rangle & =|\{8\} ; 1,-1,0\rangle & & |\Lambda\rangle=|\{8\} ; 0,0,0\rangle \\
\left|\pi^{+}\right\rangle & =-|\{8\} ; 1,1,0\rangle & & \left|\pi^{0}\right\rangle=|\{8\} ; 1,0,0\rangle \\
\left|\pi^{-}\right\rangle & =|\{8\} ; 1,-1,0\rangle, & &
\end{aligned}
$$

where the convention here is to label the mathematical states as $\left|\{\mathbf{N}\} ; I, I_{3}, Y\right\rangle$.

The next step is to calculate the transition matrix elements $\left\langle Y \pi\left|j_{\mathrm{sc}}^{\mu}\right| N\right\rangle$. To this end, it is completely necessary to express the tensor product $|Y \pi\rangle$ in the coupled basis by using the Clebsch-Gordan coefficients that can be found in Ref. [85], taking care of the signs found in some physical states of Eq. (A3). For completeness, we provide below these expressions, although we know they are straightforward.

$$
\begin{aligned}
\left|\Lambda \pi^{0}\right\rangle= & \sqrt{\frac{3}{10}}|\{27\} ; 1,0,0\rangle-\frac{1}{2}|\{10\} ; 1,0,0\rangle-\frac{1}{2}|\{\overline{10}\} ; 1,0,0\rangle+\sqrt{\frac{1}{5}}|\{8\} ; 1,0,0\rangle \\
\left|\Lambda \pi^{-}\right\rangle= & \sqrt{\frac{3}{10}}|\{27\} ; 1,-1,0\rangle-\frac{1}{2}|\{10\} ; 1,-1,0\rangle-\frac{1}{2}|\{\overline{10}\} ; 1,-1,0\rangle+\sqrt{\frac{1}{5}}|\{8\} ; 1,-1,0\rangle \\
\left|\Sigma^{+} \pi^{-}\right\rangle= & -\sqrt{\frac{1}{6}}|\{27\} ; 2,0,0\rangle-\sqrt{\frac{1}{12}}|\{10\} ; 1,0,0\rangle+\sqrt{\frac{1}{12}}|\{\overline{10}\} ; 1,0,0\rangle-\sqrt{\frac{1}{3}}\left|\left\{8^{\prime}\right\} ; 1,0,0\right\rangle \\
& +\sqrt{\frac{1}{120}}|\{27\} ; 0,0,0\rangle+\sqrt{\frac{1}{5}}|\{8\} ; 0,0,0\rangle-\sqrt{\frac{1}{8}}|\{1\} ; 0,0,0\rangle \\
\left|\Sigma^{0} \pi^{0}\right\rangle= & \sqrt{\frac{2}{3}}|\{27\} ; 2,0,0\rangle+\sqrt{\frac{1}{120}}|\{27\} ; 0,0,0\rangle+\sqrt{\frac{1}{5}}|\{8\} ; 0,0,0\rangle-\sqrt{\frac{1}{8}}|\{1\} ; 0,0,0\rangle \\
\left|\Sigma^{-} \pi^{+}\right\rangle= & -\sqrt{\frac{1}{6}}|\{27\} ; 2,0,0\rangle+\sqrt{\frac{1}{12}}|\{10\} ; 1,0,0\rangle-\sqrt{\frac{1}{12}}|\{\overline{10}\} ; 1,0,0\rangle+\sqrt{\frac{1}{3}}\left|\left\{8^{\prime}\right\} ; 1,0,0\right\rangle \\
& +\sqrt{\frac{1}{120}}|\{27\} ; 0,0,0\rangle+\sqrt{\frac{1}{5}}|\{8\} ; 0,0,0\rangle-\sqrt{\frac{1}{8}}|\{1\} ; 0,0,0\rangle \\
\left|\Sigma^{0} \pi^{-}\right\rangle= & \sqrt{\frac{1}{2}}|\{27\} ; 2,-1,0\rangle+\sqrt{\frac{1}{12}}|\{10\} ; 1,-1,0\rangle-\sqrt{\frac{1}{12}}|\{\overline{10}\} ; 1,-1,0\rangle+\sqrt{\frac{1}{3}}\left|\left\{8^{\prime}\right\} ; 1,-1,0\right\rangle \\
\left|\Sigma^{-} \pi^{0}\right\rangle= & \sqrt{\frac{1}{2}}|\{27\} ; 2,-1,0\rangle-\sqrt{\frac{1}{12}}|\{10\} ; 1,-1,0\rangle+\sqrt{\frac{1}{12}}|\{10\} ; 1,-1,0\rangle-\sqrt{\frac{1}{3}}\left|\left\{8^{\prime}\right\} ; 1,-1,0\right\rangle .
\end{aligned}
$$

Now we calculate the matrix elements $\left\langle Y \pi\left|j_{\mathrm{sc}}^{\mu}\right| N\right\rangle$ but expressing the bras $\langle Y \pi|$ in terms of the coupled basis as given in Eqs. (A4)-(A10), and then apply the Wigner-Eckart theorem to each matrix element because now we have an irreducible tensor operator between states belonging to irreducible representations of the SU(3) group. For completeness, below we write the expression of the WignerEckart theorem for SU(3), which can be also found in [101], 


$$
\begin{aligned}
& \left\langle\left\{\mu_{3}\right\} ;\left(\nu_{3}\right)\left|T_{\left(\nu_{2}\right)}^{\left\{\mu_{2}\right\}}\right|\left\{\mu_{1}\right\} ;\left(\nu_{1}\right)\right\rangle \\
& \quad=\sum_{\gamma}\left(\begin{array}{ccc}
\left\{\mu_{1}\right\} & \left\{\mu_{2}\right\} & \left\{\mu_{3}\right\}_{\gamma} \\
\left(\nu_{1}\right) & \left(\nu_{2}\right) & \left(\nu_{3}\right)
\end{array}\right)\left\langle\left\{\mu_{3}\right\}\left\|T^{\left\{\mu_{2}\right\}}\right\|\left\{\mu_{1}\right\}\right\rangle_{\gamma} .
\end{aligned}
$$

In the above expression, the indices $\mu_{i}$ refer to the irreducible representations of the $\mathrm{SU}(3)$ group, while the indices $\nu_{i}$ collectively refer to the $\left(I, I_{3}, Y\right)$ "magnetic" quantum numbers of the representation $\mu_{i}$. The factor between parentheses is precisely the $\mathrm{SU}(3)$ ClebschGordan coefficient, and finally the last term in Eq. (A11) is the reduced matrix element, which is totally independent of the "magnetic" quantum numbers. Note that, in principle, a sum over $\gamma$ has to be carried out. This amounts to sum over all the times the $\left\{\mu_{3}\right\}$ irreducible representation is contained in the tensor product $\left\{\mu_{1}\right\} \otimes\left\{\mu_{2}\right\}$. However, in our case there will not be such a sum because in the bras of Eq. (A11) there will always be a definite $\left\{\mu_{3}\right\}_{\gamma}$ representation.

After having evaluated the $\left\langle Y \pi\left|j_{\mathrm{sc}}^{\mu}\right| N\right\rangle$ matrix elements for all the cases in our study, we can write the following $7 \times 6$ matrix relating the previous matrix elements with the reduced ones,

$$
\left(\begin{array}{l}
j_{p \rightarrow \Lambda \pi^{0}}^{\mu} \\
j_{n \rightarrow \Lambda \pi^{-}}^{\mu} \\
j_{p \rightarrow \Sigma^{+} \pi^{-}}^{\mu} \\
j_{p \rightarrow \Sigma^{0} \pi^{0}}^{\mu} \\
j_{p \rightarrow \Sigma^{-} \pi^{+}}^{\mu} \\
j_{n \rightarrow \Sigma^{0} \pi^{-}}^{\mu} \\
j_{n \rightarrow \Sigma^{-} \pi^{0}}^{\mu}
\end{array}\right)=\left(\begin{array}{cccccc}
\frac{\sqrt{3}}{10} & \frac{1}{\sqrt{48}} & \frac{-1}{\sqrt{48}} & \frac{-\sqrt{3}}{10} & 0 & 0 \\
\frac{\sqrt{3}}{\sqrt{50}} & \frac{1}{\sqrt{24}} & \frac{-1}{\sqrt{24}} & \frac{-\sqrt{3}}{\sqrt{50}} & 0 & 0 \\
\frac{1}{40} & \frac{1}{12} & \frac{1}{12} & \frac{1}{10} & \frac{-1}{6} & \frac{-1}{8} \\
\frac{1}{40} & 0 & 0 & \frac{1}{10} & 0 & \frac{-1}{8} \\
\frac{1}{40} & \frac{-1}{12} & \frac{-1}{12} & \frac{1}{10} & \frac{1}{6} & \frac{-1}{8} \\
0 & \frac{-1}{\sqrt{72}} & \frac{-1}{\sqrt{72}} & 0 & \frac{1}{\sqrt{18}} & 0 \\
0 & \frac{1}{\sqrt{72}} & \frac{1}{\sqrt{72}} & 0 & \frac{-1}{\sqrt{18}} & 0
\end{array}\right)\left(\begin{array}{c}
j_{\{27\}}^{\mu} \\
j_{\{10\}}^{\mu} \\
j_{\{\overline{10}\}}^{\mu} \\
j_{\{8\}}^{\mu} \\
j_{\left\{8^{\prime}\right\}}^{\mu} \\
j_{\{1\}}^{\mu}
\end{array}\right),
$$

where $j_{N \rightarrow Y \pi}^{\mu}$ is a shorthand notation for $\left\langle Y \pi\left|j_{\mathrm{sc}}^{\mu}\right| N\right\rangle$, while $j_{\{\mathbf{N}\}}^{\mu}$ is also a shorthand notation for the reduced matrix element $\left\langle\{N\}\left\|j_{\mathrm{sc}}^{\mu}\right\|\{8\}\right\rangle$, with $j_{\mathrm{sc}}^{\mu}$ given by Eq. (A2) and $\{N\}$ is any of the irreducible representations of the $\mathrm{SU}(3)$ group appearing in the Clebsch-Gordan series of the tensor product of two octets, given in Eq. (22).

Of course, the coefficient matrix of Eq. (A12) has more rows than columns, because for these $\Delta S=-1$ weak strangeness-changing transitions there are only 6 independent matrix elements, $j_{\{\mathbf{N}\}}^{\mu}$. However, not 6 matrix elements of the left-hand side of Eq. (A12) can be chosen as truly independent, because the rank of the coefficient matrix is not 6 , it is lesser. This was expectable, because there are other independent transition matrix elements that can be driven by the weak strangeness-changing operator of Eq. (A2). These can be, for instance, the $\left\langle N^{\prime} \bar{K}\left|j_{\mathrm{sc}}^{\mu}\right| N\right\rangle$ (studied in Ref. [73]), the $\left\langle\Xi K\left|j_{\mathrm{sc}}^{\mu}\right| N\right\rangle$ (studied in Ref. [75]), or the $\left\langle Y \eta\left|j_{\text {sc }}^{\mu}\right| N\right\rangle$ matrix elements.

Indeed, the rank of the coefficient matrix of Eq. (A12) is 3. It is easy to realize that the first and second rows of this matrix are proportional. If one multiplies the second row by a factor $\frac{1}{\sqrt{2}}$, one obtains the coefficients of the first row. This indicates that only one of the $j_{p \rightarrow \Lambda \pi^{0}}^{\mu}$ or $j_{n \rightarrow \Lambda \pi^{-}}^{\mu}$ can be taken as independent. The relation between them is

$$
\left\langle\Lambda \pi^{0}\left|j_{\mathrm{sc}}^{\mu}\right| p\right\rangle=\frac{1}{\sqrt{2}}\left\langle\Lambda \pi^{-}\left|j_{\mathrm{sc}}^{\mu}\right| n\right\rangle .
$$

Due to this relation between the amplitudes for $\Lambda \pi$ production, the cross sections for $n \rightarrow \Lambda \pi^{-}$channel are twice as large than those for the $p \rightarrow \Lambda \pi^{0}$ one, as can be observed in Fig. 4.

Another easy to notice relation can be drawn by observing the last two rows of the matrix of Eq. (A12). One is the negative of the other, thus implying that

$$
\left\langle\Sigma^{0} \pi^{-}\left|j_{\mathrm{sc}}^{\mu}\right| n\right\rangle=-\left\langle\Sigma^{-} \pi^{0}\left|j_{\mathrm{sc}}^{\mu}\right| n\right\rangle .
$$

This is the reason because of the cross sections for $\Sigma \pi$ production reactions off neutrons are exactly the same, as discussed in the caption of Fig. 5, and also the flux-averaged cross sections shown in the last two rows of Table V.

Nonetheless, we have decided to take as independent strangeness-changing matrix elements $\left\langle\Lambda \pi^{-}\left|j_{\mathrm{sc}}^{\mu}\right| n\right\rangle$, $\left\langle\Sigma^{+} \pi^{-}\left|j_{\mathrm{sc}}^{\mu}\right| p\right\rangle$ and $\left\langle\Sigma^{-} \pi^{+}\left|j_{\mathrm{sc}}^{\mu}\right| p\right\rangle$. This can be done because by taking the second, third and fifth rows of the matrix in Eq. (A12), one can form a $3 \times 6$ sub-matrix with at least one $3 \times 3$ determinant different from zero, i.e., these rows are linearly independent. ${ }^{6}$ With this choice, we can express three $j_{\{\mathbf{N}\}}^{\mu}$ reduced matrix elements in terms of the above linearly independent explicit amplitudes and the other three remaining reduced matrix elements. ${ }^{7}$ The result is,

$$
\begin{aligned}
j_{\{8\}}^{\mu}= & \frac{5}{6}\left(j_{\{10\}}^{\mu}-j_{\{\overline{10\}}}^{\mu}\right)+j_{\{27\}}^{\mu}-5 \sqrt{\frac{2}{3}} j_{n \rightarrow \Lambda \pi^{-}}^{\mu} \\
j_{\left\{8^{\prime}\right\}}^{\mu}= & \frac{1}{2}\left(j_{\{10\}}^{\mu}+j_{\{1 \overline{10}\}}^{\mu}\right)+3\left(j_{p \rightarrow \Sigma^{-} \pi^{+}}^{\mu}-j_{p \rightarrow \Sigma^{+} \pi^{-}}^{\mu}\right) \\
j_{\{1\}}^{\mu}= & \frac{2}{3}\left(j_{\{10\}}^{\mu}-j_{\{10\}}^{\mu}\right)+j_{\{27\}}^{\mu}-4 \sqrt{\frac{2}{3}} j_{n \rightarrow \Lambda \pi^{-}}^{\mu} \\
& -4\left(j_{p \rightarrow \Sigma^{-} \pi^{+}}^{\mu}+j_{p \rightarrow \Sigma^{+} \pi^{-}}^{\mu}\right) .
\end{aligned}
$$

\footnotetext{
${ }^{6}$ One could have taken equally other 3 different amplitudes with the same properties of linear independence, but we have decided to make this choice.

${ }^{7}$ One cannot express the six $j_{\{\mathbf{N}\}}^{\mu}$ reduced matrix elements in terms only of the three explicit linear independent amplitudes, because there are more unknowns than linearly independent equations in the system, i.e., it is an underdetermined linear system.
} 
Finally, if we replace the expressions for $j_{\{\mathbf{N}\}}^{\mu}$ given in Eqs. (A15)-(A17) in the right-hand side of the linear system of Eq. (A12), and carry out the matrix multiplication, we obtain Eq. (A13) for the first row. And also

$$
\begin{aligned}
& \left\langle\Sigma^{0} \pi^{0}\left|j_{\mathrm{sc}}^{\mu}\right| p\right\rangle=\frac{1}{2}\left(\left\langle\Sigma^{+} \pi^{-}\left|j_{\mathrm{sc}}^{\mu}\right| p\right\rangle+\left\langle\Sigma^{-} \pi^{+}\left|j_{\mathrm{sc}}^{\mu}\right| p\right\rangle\right) \\
& \left\langle\Sigma^{0} \pi^{-}\left|j_{\mathrm{sc}}^{\mu}\right| n\right\rangle=\frac{1}{\sqrt{2}}\left(\left\langle\Sigma^{-} \pi^{+}\left|j_{\mathrm{sc}}^{\mu}\right| p\right\rangle-\left\langle\Sigma^{+} \pi^{-}\left|j_{\mathrm{sc}}^{\mu}\right| p\right\rangle\right) \\
& \left\langle\Sigma^{-} \pi^{0}\left|j_{\mathrm{sc}}^{\mu}\right| n\right\rangle=-\frac{1}{\sqrt{2}}\left(\left\langle\Sigma^{-} \pi^{+}\left|j_{\mathrm{sc}}^{\mu}\right| p\right\rangle-\left\langle\Sigma^{+} \pi^{-}\left|j_{\mathrm{sc}}^{\mu}\right| p\right\rangle\right)
\end{aligned}
$$

for the fourth, sixth, and seventh rows of Eq. (A12), respectively. Notice that the relationships given in Eqs. (A19) and (A20) are fully consistent with the relation given previously in Eq. (A14).

Finally, it is worth warning the reader that these relations between the amplitudes are exact in the SU(3) limit, but when one uses the different physical masses of the involved particles, there will be $\mathrm{SU}(3)$ or $\mathrm{SU}(2)$ breaking effects. Nonetheless, these relations can be used to check that the $\mathcal{A}_{i}^{N \rightarrow Y \pi}$ constants of the Tables III and IV satisfy them. However, one has to be careful when checking these $\mathcal{A}_{i}^{N \rightarrow Y \pi}$ constants in some Born diagrams, where there are additional factors hidden in the standard definitions of the $f_{i}^{N Y}\left(q^{2}\right)$ and $g_{1}^{N Y}\left(q^{2}\right)$ form factors of Tables I and II.
[1] L. Alvarez-Ruso et al. (NuSTEC Collaboration), Prog. Part. Nucl. Phys. 100, 1 (2018).

[2] L. Alvarez-Ruso, Y. Hayato, and J. Nieves, New J. Phys. 16, 075015 (2014).

[3] T. Katori and M. Martini, J. Phys. G 45, 013001 (2018).

[4] U. Mosel, Annu. Rev. Nucl. Part. Sci. 66, 171 (2016).

[5] U. Mosel, J. Phys. G 46, 113001 (2019).

[6] D. Drakoulakos et al. (MINERvA Collaboration), arXiv: hep-ex/0405002.

[7] K. Abe et al. (T2K Collaboration), Nucl. Instrum. Methods Phys. Res., Sect. A 659, 106 (2011).

[8] H. Chen et al. (MicroBooNE Collaboration), Fermi National Accelerator Laboratory Report No. FERMILAB-PROPOSAL-0974.

[9] K. Abe et al. (Hyper-Kamiokande Proto- Collaboration), Prog. Theor. Exp. Phys. 2015, $053 \mathrm{C} 02$ (2015).

[10] R. Acciarri et al. (DUNE Collaboration), arXiv: 1601.05471.

[11] R. Acciarri et al. (DUNE Collaboration), arXiv:1512.06148.

[12] P. Dennery, Phys. Rev. 127, 664 (1962).

[13] D. Rein and L. M. Sehgal, Ann. Phys. (N.Y.) 133, 79 (1981).

[14] C. Wilkinson, P. Rodrigues, S. Cartwright, L. Thompson, and K. McFarland, Phys. Rev. D 90, 112017 (2014).

[15] E. Hernández, J. Nieves, and M. J. Vicente Vacas, Phys. Rev. D 87, 113009 (2013).

[16] E. A. Paschos, L. Pasquali, and J. Y. Yu, Nucl. Phys. B588, 263 (2000).

[17] O. Lalakulich, T. Leitner, O. Buss, and U. Mosel, Phys. Rev. D 82, 093001 (2010).

[18] K. S. Kuzmin, V. V. Lyubushkin, and V. A. Naumov, Acta Phys. Pol. B 37, 2337 (2006).

[19] J. J. Wu, T. Sato, and T. S. H. Lee, Phys. Rev. C 91, 035203 (2015).

[20] T. Leitner, O. Buss, U. Mosel, and L. Alvarez-Ruso, Phys. Rev. C 79, 038501 (2009).

[21] M. Kabirnezhad, Phys. Rev. D 97, 013002 (2018).
[22] U. Mosel and K. Gallmeister, Phys. Rev. C 96, 015503 (2017).

[23] M. V. Ivanov, J. M. Udias, A. N. Antonov, J. A. Caballero, M. B. Barbaro, and E. M. de Guerra, Phys. Lett. B 711, 178 (2012).

[24] A. Nikolakopoulos, R. González-Jiménez, K. Niewczas, J. Sobczyk, and N. Jachowicz, Phys. Rev. D 97, 093008 (2018).

[25] C. Barbero, G. López Castro, and A. Mariano, Phys. Lett. B 728, 282 (2014).

[26] M. Martini, M. Ericson, G. Chanfray, and J. Marteau, Phys. Rev. C 80, 065501 (2009).

[27] E. Hernandez, J. Nieves, and M. Valverde, Phys. Rev. D 76, 033005 (2007).

[28] J. E. Sobczyk, E. Hernández, S. X. Nakamura, J. Nieves, and T. Sato, Phys. Rev. D 98, 073001 (2018).

[29] D. L. Yao, L. Alvarez-Ruso, A. N. Hiller Blin, and M. J. Vicente Vacas, Phys. Rev. D 98, 076004 (2018).

[30] D. L. Yao, L. Alvarez-Ruso, and M. J. Vicente Vacas, Phys. Lett. B 794, 109 (2019).

[31] L. Alvarez-Ruso, S. K. Singh, and M. J. Vicente Vacas, Phys. Rev. C 59, 3386 (1999).

[32] T. Leitner, O. Buss, L. Alvarez-Ruso, and U. Mosel, Phys. Rev. C 79, 034601 (2009).

[33] S. Ahmad, M. Sajjad Athar, and S. K. Singh, Phys. Rev. D 74, 073008 (2006).

[34] M. Rafi Alam, M. Sajjad Athar, S. Chauhan, and S. K. Singh, Int. J. Mod. Phys. E 25, 1650010 (2016).

[35] R. González-Jiménez, N. Jachowicz, K. Niewczas, J. Nys, V. Pandey, T. Van Cuyck, and N. Van Dessel, Phys. Rev. D 95, 113007 (2017).

[36] O. Lalakulich and E. A. Paschos, Phys. Rev. D 71, 074003 (2005).

[37] O. Lalakulich, E. A. Paschos, and G. Piranishvili, Phys. Rev. D 74, 014009 (2006).

[38] S. S. Gershtein, Y. Y. Komachenko, and M. Y. Khlopov, Sov. J. Nucl. Phys. 32, 861 (1980). 
[39] I. Budagov, D. C. Cundy, C. Franzinetti, W. B. Fretter, H. W. K. Hopkins, C. Manfredotti, G. Myatt, F. A. Nezrick, M. Nikolic, T. B. Novey et al., Phys. Lett. B 29, 524 (1969).

[40] G. M. Radecky, V. E. Barnes, D. D. Carmony, A. F. Garfinkel, M. Derrick, E. Fernandez, L. Hyman, G. Levman, D. Koetke, B. Musgrave et al., Phys. Rev. D 25, 1161 (1982); 26, 3297(E) (1982).

[41] T. Kitagaki, H. Yuta, S. Tanaka, A. Yamaguchi, K. Abe, K. Hasegawa, K. Tamai, S. Kunori, Y. Otani, H. Hayano et al., Phys. Rev. D 34, 2554 (1986).

[42] W. Krenz et al. (Gargamelle Neutrino Propane and Aachen-Brussels-CERN-Ecole Poly-Orsay-Padua Collaboration), Nucl. Phys. B135, 45 (1978).

[43] W. Y. Lee, E. Maddry, P. Sokolsky, L. Teig, A. Bross, T. Chapin, L. Holloway, L. Nodulman, T. O'Halloran, C. Y. Pang et al., Phys. Rev. Lett. 38, 202 (1977).

[44] H. J. Grabosch et al. (SKAT Collaboration), Z. Phys. C 41, 527 (1989).

[45] C. L. McGivern et al. (MINERvA Collaboration), Phys. Rev. D 94, 052005 (2016).

[46] Y. Kurimoto et al. (SciBooNE Collaboration), Phys. Rev. D 81, 033004 (2010).

[47] S. J. Barish, Y. Cho, M. Derrick, T. Dombeck, L. G. Hyman, B. Musgrave, P. Schreiner, R. Singer, R. P. Smith, D. Swanson et al., Phys. Rev. Lett. 36, 179 (1976).

[48] O. Erriquez, M. T. Fogli-Muciaccia, S. Natali, S. Nuzzo, A. Halsteinslid, K. Myklebost, A. Rognebakke, O. Skjeggestad, S. Bonetti, D. Cavalli et al., Phys. Lett. 73B, 350 (1978).

[49] K. Abe et al. (T2K Collaboration), Phys. Rev. D 95, 012010 (2017).

[50] T. Bolognese, J. P. Engel, J. L. Guyonnet, and J. L. Riester, Phys. Lett. 81B, 393 (1979).

[51] M. Derrick, E. Fernandez, L. Hyman, G. Levman, D. Koetke, B. Musgrave, P. Schreiner, R. Singer, A. Snyder, S. Toaff et al., Phys. Rev. D 23, 569 (1981).

[52] N. J. Baker, A. M. Cnops, P. L. Connolly, S. A. Kahn, M. J. Murtagh, R. B. Palmer, N. P. Samios, and M. Tanaka, Phys. Rev. D 23, 2495 (1981).

[53] F. J. Hasert, W. Krenz, J. Von Krogh, D. Lanske, J. Morfin, K. Schultze, H. Weerts, G. H. B. Coremans, H. Mulkens, J. Sacton et al., Phys. Lett. 59B, 485 (1975).

[54] M. Pohl, M. Dewit, C. Vander Velde-Wilquet, P. Vilain, D. Haidt, C. Matteuzzi, B. Degrange, T. Francois, P. Van Dam, M. Jaffre et al., Lett. Nuovo Cimento 24, 540 (1979).

[55] D. Allasia et al. (AMSTERDAM-BOLOGNA-PADUAPISA-SACLAY-TURIN Collaboration), Z. Phys. C 20, 95 (1983).

[56] D. Coplowe et al. (MINERvA Collaboration), Phys. Rev. D 102, 072007 (2020).

[57] A. A. Aguilar-Arevalo et al. (MiniBooNE Collaboration), Phys. Rev. D 83, 052007 (2011).

[58] B. Eberly et al. (MINERvA Collaboration), Phys. Rev. D 92, 092008 (2015).

[59] T. Le et al. (MINERvA Collaboration), Phys. Lett. B 749, 130 (2015).

[60] A. A. Aguilar-Arevalo et al. (MiniBooNE Collaboration), Phys. Rev. D 83, 052009 (2011).
[61] A. Rodriguez et al. (K2K Collaboration), Phys. Rev. D 78, 032003 (2008).

[62] X. L. Ren, E. Oset, L. Alvarez-Ruso, and M. J. Vicente Vacas, Phys. Rev. C 91, 045201 (2015).

[63] J. J. Wu and B. S. Zou, Few Body Syst. 56, 165 (2015).

[64] J. Finjord and F. Ravndal, Nucl. Phys. B106, 228 (1976).

[65] H. K. Dewan, Phys. Rev. D 24, 2369 (1981).

[66] R. P. Feynman, M. Kislinger, and F. Ravndal, Phys. Rev. D 3, 2706 (1971).

[67] S. K. Singh and M. J. Vicente Vacas, Phys. Rev. D 74, 053009 (2006).

[68] K. S. Kuzmin and V. A. Naumov, Phys. At. Nucl. 72, 1501 (2009).

[69] M. R. Alam, M. S. Athar, S. Chauhan, and S. K. Singh, J. Phys. G 42, 055107 (2015).

[70] A. Fatima, M. S. Athar, and S. K. Singh, Front. Phys. 7, 13 (2019).

[71] J. E. Sobczyk, N. Rocco, A. Lovato, and J. Nieves, Phys. Rev. C 99, 065503 (2019).

[72] M. Rafi Alam, I. Ruiz Simo, M. Sajjad Athar, and M. J. Vicente Vacas, Phys. Rev. D 82, 033001 (2010).

[73] M. R. Alam, I. R. Simo, M. S. Athar, and M. J. Vicente Vacas, Phys. Rev. D 85, 013014 (2012).

[74] M. Rafi Alam, I. Ruiz Simo, M. Sajjad Athar, and M. J. Vicente Vacas, Phys. Rev. D 87, 053008 (2013).

[75] M. Rafi Alam and I. Ruiz Simo, Phys. Rev. D 100, 033001 (2019).

[76] S. Scherer and M. R. Schindler, Lect. Notes Phys. 830, 1 (2012).

[77] V. Shtabovenko, R. Mertig, and F. Orellana, Comput. Phys. Commun. 256, 107478 (2020).

[78] V. Shtabovenko, R. Mertig, and F. Orellana, Comput. Phys. Commun. 207, 432 (2016).

[79] R. Mertig, M. Bohm, and A. Denner, Comput. Phys. Commun. 64, 345 (1991).

[80] J. Gasser and H. Leutwyler, Nucl. Phys. B250, 465 (1985).

[81] N. Cabibbo, E. C. Swallow, and R. Winston, Annu. Rev. Nucl. Part. Sci. 53, 39 (2003).

[82] S. Weinberg, Phys. Rev. 112, 1375 (1958).

[83] A. Fatima, M. Sajjad Athar, and S. K. Singh, Phys. Rev. D 98, 033005 (2018).

[84] J. A. Oller, M. Verbeni, and J. Prades, J. High Energy Phys. 09 (2006) 079.

[85] P. S. J. McNamee and F. Chilton, Rev. Mod. Phys. 36, 1005 (1964).

[86] S. Galster, H. Klein, J. Moritz, K. H. Schmidt, D. Wegener, and J. Bleckwenn, Nucl. Phys. B32, 221 (1971).

[87] F. Akbar, M. Sajjad Athar, A. Fatima, and S. K. Singh, Eur. Phys. J. A 53, 154 (2017).

[88] Y. Nambu, Phys. Rev. Lett. 4, 380 (1960).

[89] M. N. Butler, M. J. Savage, and R. P. Springer, Nucl. Phys. B399, 69 (1993).

[90] M. Doring, E. Oset, and S. Sarkar, Phys. Rev. C 74, 065204 (2006).

[91] P. A. Zyla et al. (Particle Data Group Collaboration), Prog. Theor. Exp. Phys. 2020, 083C01 (2020).

[92] E. Oset and A. Ramos, Nucl. Phys. A635, 99 (1998). 
[93] A. A. Aguilar-Arevalo et al. (MiniBooNE Collaboration), Phys. Rev. D 88, 032001 (2013).

[94] K. Hiraide et al. (SciBooNE Collaboration), Phys. Rev. D 78, 112004 (2008).

[95] K. Abe et al. (T2K Collaboration), Phys. Rev. D 87, 012001 (2013).

[96] K. Abe et al. (T2K Collaboration), Phys. Rev. D 91, 072010 (2015).

[97] L. Aliaga et al. (MINERvA Collaboration), Phys. Rev. D 94, 092005 (2016).
[98] P. Hamacher-Baumann, X. Lu, and J. Martín-Albo, Phys. Rev. D 102, 033005 (2020).

[99] X. G. Lu, D. Coplowe, R. Shah, G. Barr, D. Wark, and A. Weber, Phys. Rev. D 92, 051302 (2015).

[100] A. Faessler, T. Gutsche, B. R. Holstein, M. A. Ivanov, J. G. Korner, and V. E. Lyubovitskij, Phys. Rev. D 78, 094005 (2008).

[101] J. J. de Swart, Rev. Mod. Phys. 35, 916 (1963); 37, 326(E) (1965). 